\title{
Classes de Wadge potentielles et théorèmes d'uniformisation partielle
}

\author{
par
}

\author{
Dominique L e com te (Paris)
}

Résumé. On cherche à donner une construction aussi simple que possible d'un borélien donné d'un produit de deux espaces polonais. D'où l'introduction de la notion de classe de Wadge potentielle. On étudie notamment ce que signifie "ne pas être potentiellement fermé", en montrant des résultats de type Hurewicz. Ceci nous amène naturellement à des théorèmes d'uniformisation partielle, sur des parties "grosses", au sens du cardinal ou de la catégorie.

0. Introduction. La notion de classe de Wadge permet de mesurer la complexité topologique d'un borélien d'un espace polonais de dimension 0 . On peut se demander si la complexité d'un borélien donné ne diminue pas en raffinant la topologie polonaise de l'espace ambiant. Comme on le verra au tout début, la réponse est positive : tout borélien peut être rendu ouvert.

Mais la question analogue se pose avec des topologies produit, notamment lors de l'étude des relations d'équivalence boréliennes; d'où l'introduction de la notion de classe de Wadge potentielle (on détaillera ce lien dans le chapitre 1). Après avoir effectué quelques rappels et établi quelques propriétés de base, on donne une nouvelle démonstration d'un premier résultat d'uniformisation partielle, déjà prouvé par Przymusi/nski : une condition nécessaire et suffisante pour qu'un borélien contienne un graphe de fonction borélienne à image non dénombrable. Ensuite, on verra qu'à chaque niveau de complexité on peut trouver un borélien dont la complexité ne diminue pas.

Suite à quoi on cherchera à savoir si certains résultats vrais pour les classes de Wadge peuvent être adaptés aux classes de Wadge potentielles. En l'occurrence, il s'agit d'une part de voir si cette notion correspond à une réduction, comme dans le cas des classes de Wadge classiques, et on verra que non. On cherchera ensuite à savoir si on peut obtenir des résultats de type Hurewicz, c'est-à-dire : ne pas être d'une classe donnée, c'est être au

1991 Mathematics Subject Classification: Primary 04A15. 
moins aussi compliqué que des exemples de référence n'étant pas de cette classe.

On obtiendra des résultats partiels pour les potentiellement fermés, et pour les petites classes de Wadge (notamment, on caractérisera les boréliens potentiellement fermés parmi les boréliens à coupes dénombrables, à l'aide d'ensembles localement à projections ouvertes).

Ce qui nous amènera à de nouveaux résultats d'uniformisation, pour des ensembles à coupes maigres et des $G_{\delta}$ denses, dont le but est d'obtenir d'autres caractérisations que celles évoquées ci-dessus.

1. Notations et rappels. On utilisera les notations standard de la théorie descriptive des ensembles, qui peuvent être trouvées dans [Mo]. Par exemple, on notera $D_{2}\left(\boldsymbol{\Sigma}_{1}^{0}\right)$ la classe des différences de deux ouverts.

$\Gamma$ désignera une famille d'ensembles, et $\Gamma\lceil X$ les parties de $X$ qui sont dans $\Gamma$. Par exemple, $\boldsymbol{\Delta}_{1}^{1}\left\lceil\mathbf{2}^{\omega}\right.$ désignera l'ensemble des boréliens de $\mathbf{2}^{\omega}$.

Dans un espace polonais récursivement présenté, $\Sigma$ désignera la topologie engendrée par les $\Sigma_{1}^{1}$ (c'est la topologie de Gandy-Harrington), $\Delta$ la topologie engendrée par les $\Delta_{1}^{1}$, ce qu'on pourra noter aussi $\prec \Delta_{1}^{1} \succ$ (cette topologie est polonaise : cf. [Lo4]).

Si $X$ est un tel espace, on pose $\Omega_{X}:=\left\{x \in X: \omega_{1}^{x}=\omega_{1}^{\mathrm{CK}}\right\}$. Rappelons (cf. [Mo]) que $\Omega_{X}$ est $\Sigma_{1}^{1}$, et un $\Sigma$-ouvert dense (cf. [Lo1]). Les traces des $\Sigma_{1}^{1}$ sur $\Omega_{X}$ sont $\boldsymbol{\Delta}_{1}^{0}\left\lceil\left(\Omega_{X}, \Sigma\left\lceil\Omega_{X}\right)\right.\right.$; en effet, si $A$ est $\Sigma_{1}^{1}$ contenu dans $\Omega_{X}$ et $f$ est $\Delta_{1}^{1}$ telle que $f(x) \in \mathrm{WO} \Leftrightarrow x \notin A$, on a

$$
x \notin A \Leftrightarrow x \notin \Omega_{X} \text { ou }\left[x \in \Omega_{X} \text { et } \exists \xi<\omega_{1}^{\mathrm{CK}}(f(x) \in \mathrm{WO} \text { et }|f(x)| \leq \xi)\right] .
$$

L'espace $\left(\Omega_{X}, \Sigma\left\lceil\Omega_{X}\right)\right.$ est donc à base dénombrable d'ouverts-fermés, donc métrisable séparable; on sait (cf. [Lo1]) que c'est un espace fortement $\alpha$ favorable, donc c'est un espace polonais, de dimension 0 par ce qui précède.

Si $X$ et $Y$ sont des espaces topologiques, $\Pi_{X}\left(\right.$ resp. $\left.\Pi_{Y}\right)$ désignera la projection de $X \times Y$ sur $X$ (resp. $Y$ ). Le symbole $\delta(C)$ désignera le diamètre de $C$ pour une distance qui rende complet l'espace polonais ambiant.

Par ailleurs, je renvoie le lecteur à $[\mathrm{Ku}]$ et $[\mathrm{Mo}]$ pour ce qui concerne les notions de base en topologie, en théorie descriptive et en théorie effective, et à $[\mathrm{W}]$ et [Lo2] pour les résultats sur les classes de Wadge, qui seront rappelés en cas de besoin. Rappelons tout de même certains de ces résultats.

Soit $P_{0}$ (resp. $P$ ) un espace polonais de dimension 0, et $A_{0}$ (resp. $A$ ) un borélien de $P_{0}$ (resp. $P$ ). On dira que $A$ est dans la classe de Wadge engendrée par $A_{0}$ (notée $\left\langle A_{0}\right\rangle$ ) s'il existe une fonction continue $f$ de $P$ dans $P_{0}$ telle que $A=f^{-1}\left(A_{0}\right)$.

Si $\Gamma$ est une famille d'ensembles, on notera $\check{\Gamma}:=\{\check{A}: A \in \Gamma\}$. Par exemple, $\check{\Sigma}_{1}^{0}=\boldsymbol{\Pi}_{1}^{0}$. On dira que $\Gamma$ est auto-duale si $\Gamma=\check{\Gamma}$. 
Soit $\Gamma$ une famille de boréliens d'espaces polonais de dimension 0 , stable par image réciproque continue. On montre que $\Gamma$ est une classe de Wadge non auto-duale si et seulement si $\Gamma$ admet un universel. Ainsi les classes de Baire additives et multiplicatives sont des classes de Wadge non auto-duales, par exemple.

On ordonne les classes de Wadge par l'inclusion; ce qui précède montre que cet ordre n'est pas total : deux classes de Wadge non auto-duales duales l'une de l'autre sont incomparables. On a cependant le théorème de Wadge : si $\Gamma_{1}$ et $\Gamma_{2}$ sont des classes de Wadge, alors $\Gamma_{1} \subseteq \Gamma_{2}$ ou $\Gamma_{2} \subseteq \check{\Gamma}_{1}$. Cet ordre est également bien fondé. On sait aussi qu'une classe de Wadge non auto-duale a pour successeur une classe de Wadge auto-duale, et qu'une classe de Wadge auto-duale a pour successeurs deux classes de Wadge non auto-duales duales l'une de l'autre.

On montre également que si $\Gamma$ est non auto-duale, $\mathbf{2}^{\omega}$ contient un vrai $\Gamma$, c'est-à-dire un élément de $\Gamma \backslash \check{\Gamma}$.

Si $F$ est fermé dans l'espace polonais $P$ de dimension 0 , dire que $A$ est dans $\Gamma\lceil F$ équivaut à affirmer l'existence de $B$ dans $\Gamma\lceil P$ tel que $A=B \cap F$.

Enfin, si $\xi$ est un ordinal dénombrable non nul, on note $\boldsymbol{\Delta}_{\xi}^{0} \mathrm{PU}(\Gamma)$ la classe suivante : $\left\{\bigcup_{n \in \omega} A_{n} \cap U_{n}:\left(U_{n}\right) \boldsymbol{\Delta}_{\xi}^{0}\right.$-partition, $\left.A_{n} \in \Gamma\right\}$. On montre que si $\Gamma$ est une classe de Wadge, $\Gamma=\boldsymbol{\Delta}_{1}^{0} \mathrm{PU}(\Gamma)$, et que si de plus $\Gamma \neq\{\emptyset\}$ et $\check{\Gamma} \neq\{\emptyset\}$, il existe un plus grand ordinal dénombrable $\xi$ tel que $\Gamma=\boldsymbol{\Delta}_{\xi}^{0} \mathrm{PU}(\Gamma)$, appelé niveau de $\Gamma$ (on convient que $\{\emptyset\}$ et $\{\emptyset\}^{\vee}$ sont de niveau 0$)$.

RAPPEL 1.1. (a) $[\mathrm{Ku}]$ Si $X$ est un espace polonais et $\left(B_{n}\right)$ une suite de boréliens de $X$, il existe une topologie polonaise sur $X$, plus fine que la topologie initiale, rendant les $B_{n}$ ouverts.

(b) [Lo3] $S i(X, \sigma)$ et $Y$ sont des espaces polonais et $B$ un borélien de $X \times Y$ ayant ses coupes verticales $\boldsymbol{\Sigma}_{\xi}^{0}$, il existe une topologie polonaise $\sigma^{\prime}$ sur $X$, plus fine que $\sigma$, telle que $B$ soit $\Sigma_{\xi}^{0}$ dans $\left(X, \sigma^{\prime}\right) \times Y$.

LEMME 1.2. Soit $(X, \sigma)$ un espace polonais; alors il existe une topologie polonaise de dimension 0 sur $X$ plus fine que $\sigma$.

Démonstration. Soit $\left(U_{n}^{0}\right)$ une base de la topologie de $\sigma_{0}:=\sigma$. A l'aide du rappel 1.1(a), on trouve une topologie $\sigma_{1}$ rendant fermés les $U_{n}^{0}$. Soit $\left(U_{n}^{1}\right)$ une base de $\sigma_{1}$. On construit comme ceci par récurrence une suite croissante $\left(\sigma_{n}\right)$ de topologies polonaises sur $X$ telle que si $\left(U_{n}^{p}\right)_{n \in \omega}$ est une base de $\sigma_{p}, U_{n}^{p}$ est fermé de $\left(X, \sigma_{p+1}\right)$. Posons $S_{n}:=\left\{U_{p}^{j}: j \leq n, p \in \omega\right\}$; alors $\sigma_{n}=\prec S_{n} \succ$, et $\bigcap_{n \in \omega} S_{n}=S_{0}$, donc $\sigma^{\prime}:=\prec \bigcup_{n \in \omega} S_{n} \succ$ répond au problème : $\left(X, \sigma^{\prime}\right)$ est homéomorphe à la diagonale de $\prod_{n \in \omega}\left(X, \sigma_{n}\right)$, qui est fermée dans $\prod_{n \in \omega}\left(X, \sigma_{n}\right)$. 
Proposition 1.3. Si $X$ est un espace polonais et $\left(B_{n}\right)$ une suite de boréliens de $X$, il existe une topologie polonaise de dimension 0 sur $X$, plus fine que la topologie initiale, rendant les $B_{n}$ ouverts.

Démonstration. On applique le rappel 1.1(a) et le lemme 1.2.

DÉfinition 1.4. Soient $X$ et $Y$ des espaces polonais, et $A$ un borélien de $X \times Y$. Si $\Gamma$ est une classe de Wadge de boréliens, on dira que $A$ est potentiellement dans $\Gamma$ (ce qu'on notera $A \in \operatorname{Pot}(\Gamma)$ ) s'il existe des topologies polonaises de dimension $0, \sigma(\operatorname{sur} X)$ et $\tau(\operatorname{sur} Y)$, plus fines que les topologies initiales, telles que $A$, considéré comme partie de $(X, \sigma) \times$ $(Y, \tau)$, soit dans $\Gamma$.

Dans l'étude des relations d'équivalence boréliennes, par exemple dans [HKL], on étudie le pré-ordre qui suit. Si $E$ (resp. $E^{\prime}$ ) est une relation d'équivalence borélienne sur l'espace polonais $X$ (resp. $X^{\prime}$ ), on pose

$$
E \leq E^{\prime} \Leftrightarrow\left(\exists f: X \rightarrow X^{\prime} \text { borélienne telle que } x E y \Leftrightarrow f(x) E^{\prime} f(y)\right) .
$$

La dernière relation peut s'écrire : $E=(f \times f)^{-1}\left(E^{\prime}\right)$; or si $E^{\prime}$ est dans $\Gamma$ (ou même si $E^{\prime}$ est $\left.\operatorname{Pot}(\Gamma)\right)$ et $E=(f \times f)^{-1}\left(E^{\prime}\right), E$ est $\operatorname{Pot}(\Gamma)$ : si $\left(U_{n}\right)$ et $\left(V_{n}\right)$ sont des bases des topologies associées à $E^{\prime}$, on peut rendre les $f^{-1}\left(U_{n}\right)$ et les $f^{-1}\left(V_{n}\right)$ ouverts, par la proposition 1.3, ce qui fournit une topologie $\sigma$; on a alors $E \in \Gamma\lceil(X, \sigma) \times(X, \sigma)$. Ceci motive l'introduction de la notion de classe de Wadge potentielle.

Remarque 1.5. (a) Si $A$ est un borélien à coupes verticales $\Sigma_{\xi}^{0}$ d'un produit de deux espaces polonais, alors $A$ est $\operatorname{Pot}\left(\boldsymbol{\Sigma}_{\xi}^{0}\right)$.

En effet, on applique le rappel 1.1(b) et le lemme 1.2.

Remarque 1.5. (b) [Lo4] Si $\Gamma$ est une classe de Wadge et $B$ est $\Delta_{1}^{1}(\alpha)$ dans $\omega^{\omega} \times \omega^{\omega}$, alors $B$ est $\operatorname{Pot}(\Gamma)$ si et seulement si $B$, considéré comme partie de $\omega^{\omega} \times \omega^{\omega}$ muni de la topologie $\Delta_{\alpha}^{2}$, est dans $\Gamma$.

Rappelons enfin deux résultats sur les ensembles maigres.

Proposition 1.6 [Lo1]. Si $X$ est un espace polonais parfait non vide et $A$ un sous-ensemble maigre de $X \times X$, on trouve une copie $P$ de $\mathbf{2}^{\omega}$ dans $X$ telle que si $x$ et $y$ sont distincts dans $P,(x, y)$ n'est pas dans $A$.

Conollaire 1.7. Si $X$ et $Y$ sont des espaces polonais parfaits non vides et $A$ un sous-ensemble maigre de $X \times Y$, on trouve une copie $P$ (resp. $Q$ ) de $\mathbf{2}^{\omega}$ dans $X$ (resp. $Y$ ) telles que $(P \times Q) \cap A=\emptyset$.

Démonstration. On peut supposer que $X, Y=\omega^{\omega}$; en effet, si $\left(U_{n}\right)$ est une base de la topologie de $X, X^{\prime}:=X \backslash \bigcup_{n \in \omega}\left(\bar{U}_{n} \backslash U_{n}\right)$ est $G_{\delta}$ dense de $X$, donc polonais parfait, et est de dimension 0 . Soit $\left(x_{n}\right)$ une suite dense de $X^{\prime}$; alors $X^{\prime \prime}:=X^{\prime} \backslash\left\{x_{n}: n \in \omega\right\}$ est $G_{\delta}$ dense de $X^{\prime}$, donc polonais de 
dimension 0 , et est localement non compact, donc homéomorphe à $\omega^{\omega}$. De même avec $Y$.

On applique alors la proposition 1.6 à $X=Y=\omega^{\omega}$; ceci fournit une injection continue $\psi$ de $\mathbf{2}^{\omega}$ dans $\omega^{\omega}$ dont l'image est le $P$ de la proposition 1.6; on peut alors poser $P:=\psi^{\prime \prime} N_{(0)}$ et $Q:=\psi^{\prime \prime} N_{(1)}$.

\section{Ensembles potentiellement ouverts}

Rem arque 2.1. Soit $A$ un borélien d'un produit de deux espaces polonais. Si $A$ a une de ses projections dénombrable, donc en particulier si $A$ est dénombrable, $A$ est $\operatorname{Pot}\left(\boldsymbol{\Delta}_{1}^{0}\right)$.

En effet, si par exemple $\Pi_{X}^{\prime \prime} A$ est dénombrable, on rend les coupes verticales de $A$ ouvertes-fermées, par la proposition 1.3, de sorte que $A$ est $\operatorname{Pot}\left(\boldsymbol{\Sigma}_{1}^{0}\right) \cap \operatorname{Pot}\left(\boldsymbol{\Pi}_{1}^{0}\right)$, par la remarque 1.5(a). Ceci fournit des topologies $\sigma$ et $\sigma^{\prime}$ sur $X$, et $\tau$ et $\tau^{\prime}$ sur $Y$, de bases respectives $\left(U_{n}\right),\left(U_{n}^{\prime}\right),\left(V_{n}\right),\left(V_{n}^{\prime}\right)$.

On remarque ensuite que si $(Z, \mu)$ est polonais et $\mu^{\prime}$ est polonaise sur $Z$ et plus fine que $\mu$, l'application identique, de $\left(Z, \mu^{\prime}\right)$ dans $(Z, \mu)$, est bijective continue; son inverse est donc borélienne, et par conséquent, les boréliens de $(Z, \mu)$ et $\left(Z, \mu^{\prime}\right)$ coïncident.

On applique alors la proposition 1.3 à $X,\left(U_{n}\right)$ et $\left(U_{n}^{\prime}\right)$, qui sont des boréliens de $X$, d'une part, et à $Y,\left(V_{n}\right)$ et $\left(V_{n}^{\prime}\right)$ d'autre part, pour avoir le résultat.

Ceci montre en particulier que la notion de classe de Wadge potentielle n'a d'intérêt que si les espaces polonais ambiants sont non dénombrables.

Proposition 2.2. Soit A un borélien d'un produit de deux espaces polonais. Alors $A$ est $\operatorname{Pot}\left(\boldsymbol{\Sigma}_{1}^{0}\right)$ si et seulement si $A$ est réunion dénombrable de rectangles boréliens.

Démonstration. Si $A$ est $\operatorname{Pot}\left(\boldsymbol{\Sigma}_{1}^{0}\right)$, on utilise la remarque de la preuve précédente. Inversement, on applique la proposition 1.3.

Il résulte de ceci que tous les boréliens d'un produit ne peuvent être rendus ouverts en conservant une topologie produit de topologies polonaises; par exemple, la diagonale $\Delta\left(\mathbf{2}^{\omega}\right)$ de $\mathbf{2}^{\omega} \times \mathbf{2}^{\omega}$ n'est pas réunion dénombrable de rectangles, donc $\Delta\left(\mathbf{2}^{\omega}\right)$ n'est pas $\operatorname{Pot}\left(\boldsymbol{\Sigma}_{1}^{0}\right)$.

On donne maintenant une nouvelle preuve du premier théorème d'uniformisation partielle annoncé; assez curieusement, la discussion s'articule autour de la notion d'ensembles potentiellement ouverts. Ce théorème sera en outre appliqué au chapitre 3 . Notons que ce théorème a déjà été démontré dans $[\mathrm{P}]$.

ThÉorÈme 2.3. Soit A un borélien d'un produit de deux espaces polonais. Alors A contient un graphe de fonction injective continue définie sur une 
copie de $\mathbf{2}^{\omega}$ si et seulement si A n'est pas réunion dénombrable de rectangles boréliens dont l'un des côtés est un singleton.

Démonstration. Si $A$ contient un graphe comme dans l'énoncé, raisonnons par l'absurde : $A=\bigcup_{n \in \omega} A_{n} \times B_{n}$, avec $A_{n}$ ou $B_{n}$ singleton; si $\operatorname{Gr}(f) \subseteq A, \operatorname{Gr}(f)$ s'écrit $\bigcup_{n \in \omega}\left[\operatorname{Gr}\left(f\left\lceil A_{n}\right) \cap\left(X \times B_{n}\right)\right]\right.$; comme $\operatorname{Gr}(f)$ est non dénombrable, l'un des ensembles $\operatorname{Gr}\left(f\left\lceil A_{n}\right) \cap\left(X \times B_{n}\right)\right.$ est non dénombrable, ce qui contredit l'injectivité de $f$.

Montrons la réciproque.

Premier cas : $A$ est $\operatorname{Pot}\left(\boldsymbol{\Sigma}_{1}^{0}\right)$. D'après la proposition 2.2, on a $A=\bigcup_{n \in \omega} A_{n} \times B_{n}$, avec $A_{n}$ et $B_{n}$ boréliens, donc on peut trouver $n$ tel que $A_{n}$ et $B_{n}$ soient non dénombrables, donc boréliennement isomorphes, disons par $\varphi$. $A_{n}$ contient une copie de $\mathbf{2}^{\omega}$, qui contient un $G_{\delta}$ dense $G$ sur lequel $\varphi$ est continue; $G$ étant non dénombrable contient une copie de $\mathbf{2}^{\omega}$, d'où le résultat.

$$
\begin{aligned}
& \text { Second cas : } A \text { n'est pas } \operatorname{Pot}\left(\Sigma_{1}^{0}\right) \text {. Posons } \\
& \qquad E:=\{x \in X: A(x) \text { est dénombrable }\} .
\end{aligned}
$$

Si $E$ est co-dénombrable, $(E \times Y) \cap A$ est borélien à coupes dénombrables, donc est réunion dénombrable de graphes boréliens (par le théorème de Lusin : cf. [Mo]). De plus, par la remarque $2.1,(\check{E} \times Y) \cap A$ est $\operatorname{Pot}\left(\boldsymbol{\Delta}_{1}^{0}\right)$, donc $(E \times Y) \cap A$ n'est pas $\operatorname{Pot}\left(\boldsymbol{\Sigma}_{1}^{0}\right)$, et l'un des graphes n'est pas $\operatorname{Pot}\left(\boldsymbol{\Sigma}_{1}^{0}\right)$ (qui est stable par réunion dénombrable par la proposition 2.2). Par suite, la fonction correspondante est à image non dénombrable, par la remarque 2.1. On va alors trouver un parfait du domaine de la fonction sur lequel elle est injective, et on conclut comme au premier cas.

Si $E$ n'est pas co-dénombrable, comme il est co-analytique, $\check{E}$ contient une copie de $\mathbf{2}^{\omega}$; il suffit donc de voir que si $X=\mathbf{2}^{\omega}$ et $A$ est à coupes verticales non dénombrables, $A$ contient un graphe comme dans l'énoncé.

Posons donc

$$
F:=\left\{y \in Y: A(y) \text { est maigre dans } \mathbf{2}^{\omega}\right\} .
$$

Si $F$ est co-dénombrable, $\left(\mathbf{2}^{\omega} \times F\right) \cap A$ est borélien à coupes non dénombrables (donc non vides), donc est uniformisable par une fonction Baire-mesurable définie sur $\mathbf{2}^{\omega}$ (par le théorème de von Neumann). Cette fonction $f$ est continue sur un $G_{\delta}$ dense $G$ de $2^{\omega}$, et $f^{\prime \prime} G$ est non dénombrable : sinon, comme $G=\bigcup_{\beta \in f^{\prime \prime} G} G \cap f^{-1}(\{\beta\})$, l'un des $G \cap f^{-1}(\{\beta\})$ serait non maigre et contenu dans $A(\beta)$, ce qui contredirait le fait que $\beta$ est dans $F$. On conclut alors comme avant.

Si $F$ n'est pas co-dénombrable, comme il est borélien, $\check{F}$ contient une copie de $\mathbf{2}^{\omega}$; il suffit donc de voir que si $X=Y=\mathbf{2}^{\omega}$ et $A$ est borélien à coupes non maigres, $A$ contient un graphe comme dans l'énoncé. 
Mais par le théorème de Kuratowski-Ulam, $A$ est non maigre, donc on peut trouver $s$ et $t$ dans $2^{<\omega}$ telles que $\left(N_{s} \times N_{t}\right) \backslash A$ soit maigre. On trouve alors, par le corollaire 1.7, deux copies $P$ et $Q$ de $\mathbf{2}^{\omega}$ telles que $P \times Q \subseteq\left(N_{s} \times N_{t}\right) \cap A$, et si $\varphi$ est un homéomorphisme de $P \operatorname{sur} Q, \operatorname{Gr}(\varphi)$ répond à la question.

3. Classe de Wadge potentielle d'un borélien. On cherche maintenant à diminuer au maximum la complexité d'un borélien donné d'un produit d'espaces polonais.

Proposition et DÉfinition 3.1. Soit $A$ un borélien d'un produit d'espaces polonais. Il existe une unique classe de Wadge de boréliens, appelée classe de Wadge potentielle de $A$, et notée $\Gamma_{A}$, telle que:

(i) $A \in \operatorname{Pot}\left(\Gamma_{A}\right)$,

(ii) si $\Gamma$ est une classe de Wadge strictement contenue dans $\Gamma_{A}$, alors $A \notin \operatorname{Pot}(\Gamma)$.

Démonstration. Montrons l'existence d'une telle classe, en raisonnant par l'absurde : si $\Gamma_{0}$ est la classe de Wadge $\langle A\rangle$ engendrée par $A, A$ est $\operatorname{Pot}\left(\Gamma_{0}\right)$ et on trouve $\Gamma_{1} \psi \Gamma_{0}$ telle que $A$ soit $\operatorname{Pot}\left(\Gamma_{1}\right)$. Par récurrence, on construit comme ceci $\Gamma_{n+1} \psi \Gamma_{n}$ telles que $A$ soit $\operatorname{Pot}\left(\Gamma_{n+1}\right)$. Mais ceci contredit la bonne fondation de l'ordre de Wadge.

Montrons l'unicité d'une telle classe : si $\Gamma_{1}$ et $\Gamma_{2}$ vérifient (i), (ii) et $\Gamma_{1} \neq \Gamma_{2}, \Gamma_{1} \nsubseteq \Gamma_{2}\left(\operatorname{sinon} \Gamma_{1} \psi \Gamma_{2}\right.$ et $A$ n'est pas $\left.\operatorname{Pot}\left(\Gamma_{1}\right)\right)$, donc $\check{\Gamma}_{2} \subseteq \Gamma_{1}$, et de même $\check{\Gamma}_{1} \subseteq \Gamma_{2}$, donc $\Gamma_{1}=\check{\Gamma}_{2}$ est non auto-duale.

L'ensemble $A$ est dans $\operatorname{Pot}\left(\Gamma_{1}\right) \cap \operatorname{Pot}\left(\Gamma_{2}\right)$, donc comme dans la preuve de la remarque 2.1, on trouve des topologies $\sigma$ et $\tau$ telles que $A$ soit dans $\left(\Gamma_{1} \cap \Gamma_{2}\right)\lceil(X, \sigma) \times(Y, \tau)$. Mais la classe de Wadge $\Gamma$ engendrée par $A$, considéré comme partie de $(X, \sigma) \times(Y, \tau)$, vérifie $A \in \operatorname{Pot}(\Gamma)$ et $\Gamma \subseteq \Gamma_{1} \cap \check{\Gamma}_{1}$ $\psi \Gamma_{1}$, une contradiction.

Toute classe de Wadge potentielle est donc une classe de Wadge; on peut se demander s'il y a une réciproque. L'exemple de la diagonale de $\mathbf{2}^{\omega}$ montre que c'est vrai pour la classe des fermés, et on va voir que c'est vrai en général. On peut même préciser ce résultat, en trouvant un $\operatorname{Pot}(\Gamma)$ "maximal"; mais pour ce faire on introduit une classe de fonctions qui est le candidat naturel pour le problème de la réduction évoqué dans l'introduction, comme le montre le lemme suivant.

Soit donc $C_{0}$ la classe des fonctions telles que l'image réciproque d'un $\operatorname{Pot}\left(\boldsymbol{\Sigma}_{1}^{0}\right)$ soit aussi $\operatorname{Pot}\left(\boldsymbol{\Sigma}_{1}^{0}\right)$.

Lemme 3.2. Soient $X, Y, X^{\prime}, Y^{\prime}$ des espaces polonais, $A$ (resp. B) un borélien de $X \times Y\left(\right.$ resp. $\left.X^{\prime} \times Y^{\prime}\right)$; si f, de $X^{\prime} \times Y^{\prime}$ dans $X \times Y$, est dans $C_{0}$ et réduit $B$ à $A$, alors $\Gamma_{B}$ est contenue dans $\Gamma_{A}$. 
Démonstration. $A \in \operatorname{Pot}\left(\Gamma_{A}\right)$, ce qui fournit $\sigma$ et $\tau$ telles que $A$ est dans $\Gamma_{A}\left\lceil(X, \sigma) \times(Y, \tau)\right.$. Soient $\left(U_{n}\right)$ et $\left(V_{n}\right)$ des bases de $\sigma$ et $\tau$. Comme $f$ est dans $C_{0}$, par la proposition 2.2 on trouve des boréliens $U_{m}^{n, p}$ et $V_{m}^{n, p}$ tels que $f^{-1}\left(U_{n} \times V_{p}\right)=\bigcup_{m \in \omega} U_{m}^{n, p} \times V_{m}^{n, p}$. Par la proposition 1.3, on obtient des topologies $\sigma^{\prime}$ et $\tau^{\prime}$ rendant les $U_{m}^{n, p}$ et les $V_{m}^{n, p}$ ouverts, de sorte que $f$, de $\left(X^{\prime}, \sigma^{\prime}\right) \times\left(Y^{\prime}, \tau^{\prime}\right)$ dans $(X, \sigma) \times(Y, \tau)$, est continue. Donc $B$ est dans $\Gamma_{A}\left\lceil\left(X^{\prime}, \sigma^{\prime}\right) \times\left(Y^{\prime}, \tau^{\prime}\right)\right.$ et $B \in \operatorname{Pot}\left(\Gamma_{A}\right)$, d'où le résultat : sinon $\Gamma_{A} \subseteq \check{\Gamma}_{B}$, et $B \in \operatorname{Pot}\left(\Gamma_{B}\right) \cap \operatorname{Pot}\left(\check{\Gamma}_{B}\right)$; donc comme dans la preuve de la proposition 3.1, et par abus de langage, $B \in \operatorname{Pot}\left(\Gamma_{B} \cap \check{\Gamma}_{B}\right)$ et $\Gamma_{B}=\check{\Gamma}_{B}$; d'où $\Gamma_{A} \boldsymbol{\psi} \Gamma_{B}$, ce qui contredit $B \in \operatorname{Pot}\left(\Gamma_{A}\right)$.

ThÉORÈme 3.3. Si $\Gamma$ est une classe de Wadge de boréliens il existe $B$ dans $\Gamma\left\lceil\omega^{\omega} \times \omega^{\omega}\right.$ tel que:

(i) $\Gamma_{B}=\Gamma$,

(ii) $A$ est $\operatorname{Pot}(\Gamma)$ si et seulement s'il existe $f$ dans $C_{0}$ injective telle que $A=f^{-1}(B)$.

Démonstration. Premier cas : $\Gamma$ est non auto-duale. Soit $U$ un universel pour $\Gamma\left\lceil\omega^{\omega}, U \subseteq \omega^{\omega} \times \omega^{\omega}\right.$. Posons $(\alpha)_{i}(n):=\alpha(2 n+i)$, où $i=0,1$,

$$
\langle\gamma, \beta\rangle(n):= \begin{cases}\gamma(k) & \text { si } n=2 k, \\ \beta(k) & \text { si } n=2 k+1,\end{cases}
$$

et $B(\alpha, \beta) \Leftrightarrow U\left((\alpha)_{0},\left\langle(\alpha)_{1}, \beta\right\rangle\right)$.

Alors $B$ est aussi universel pour $\Gamma\left[\omega^{\omega}:(\cdot)_{1}\right.$ est continue, donc si $C$ est dans $\Gamma\left\lceil\omega^{\omega},(\cdot)_{1}^{-1}(C)\right.$ aussi, et il existe $\alpha$ dans $\omega^{\omega}$ tel que $(\cdot)_{1}^{-1}(C)=U_{\alpha}$; $\left\langle\alpha, 0^{\omega}\right\rangle$ est donc un code pour $C$.

$B$ est dans $\Gamma$, donc comme à la fin de la preuve du lemme précédent, $\Gamma_{B} \subseteq \Gamma$. Raisonnons par l'absurde pour montrer (i) : $\Gamma_{B} \psi \Gamma$, donc $\Gamma_{B} \subseteq \check{\Gamma} ; B$ est $\operatorname{Pot}\left(\Gamma_{B}\right)$, ce qui fournit des topologies $\sigma$ et $\tau$ telles que $B \in \Gamma_{B}\left\lceil\left(\omega^{\omega}, \sigma\right) \times\left(\omega^{\omega}, \tau\right)\right.$, et on a $B \in \check{\Gamma}\left\lceil\left(\omega^{\omega}, \sigma\right) \times\left(\omega^{\omega}, \tau\right)\right.$.

L'application identique, de $\left(\omega^{\omega}, \sigma\right)$ dans $\omega^{\omega}$, est bijective continue, donc d'inverse borélienne; son inverse est donc continue sur un $G_{\delta}$ dense $G$ de $\omega^{\omega}$; sur $G, \sigma$ et la topologie usuelle coïncident. $G$ étant non dénombrable contient une copie $L$ de $\mathbf{2}^{\omega}$, et comme $\Gamma \neq \check{\Gamma}$, on peut trouver $D$ dans $(\Gamma \backslash \check{\Gamma})\left\lceil L ; D=E \cap L\right.$, où $E \in \Gamma\left\lceil\omega^{\omega}\right.$. B étant universel, soit $\alpha$ dans $\omega^{\omega}$ tel que $B_{\alpha}=E$. Tout comme $B_{\alpha}, E$ est dans $\check{\Gamma}\left\lceil\left(\omega^{\omega}, \sigma\right)\right.$, donc $E \cap G$ est dans $\check{\Gamma}\lceil G$ car sur $G$, les topologies sont identiques. Donc $D$ est dans $\check{\Gamma}\lceil L$, une contradiction qui montre que $\Gamma=\Gamma_{B}$.

Pour (ii), si $A=f^{-1}(B), A$ est $\operatorname{Pot}(\Gamma)$ à cause du lemme précédent. Inversement, si $A$ est $\operatorname{Pot}(\Gamma)$, on trouve $\sigma^{\prime}$ et $\tau^{\prime}$ telles que $A$ soit dans $\Gamma\left\lceil\left(X, \sigma^{\prime}\right) \times\left(Y, \tau^{\prime}\right)\right.$. On trouve des fermés $F$ et $H$ de $\omega^{\omega}$, et des homéomorphismes : $\varphi$, de $\left(X, \sigma^{\prime}\right)$ sur $F$, et $\psi$, de $\left(Y, \tau^{\prime}\right)$ sur $H .(\varphi \times \psi)^{\prime \prime} A \in \Gamma\lceil(F \times H)$, donc est la trace sur $F \times H$ de $R \in \Gamma\left\lceil\omega^{\omega} \times \omega^{\omega} .\langle\cdot, \cdot\rangle\right.$ est un homéomorphisme, 
donc il existe $\alpha$ dans $\omega^{\omega}$ tel que $\langle\cdot, \cdot\rangle^{\prime \prime} R=U_{\alpha}$, ce qui s'écrit

$$
R(\gamma, \beta) \Leftrightarrow U(\alpha,\langle\gamma, \beta\rangle) \Leftrightarrow B(\langle\alpha, \gamma\rangle, \beta) .
$$

La fonction $f:=g \circ(\varphi \times \psi) \circ$ Id répond à la question, si on pose

$$
g: F \times H \rightarrow \omega^{\omega} \times \omega^{\omega}, \quad(\gamma, \beta) \mapsto(\langle\alpha, \gamma\rangle, \beta),
$$

puisque par la proposition 2.2, les fonctions de la forme $u \times v$, avec $u$ et $v$ boréliennes, sont dans $C_{0}$.

Second cas : $\Gamma$ est auto-duale. On sait qu'alors: ou bien il existe une suite strictement croissante $\left(\Gamma_{n}\right)$, cofinale dans $\Gamma$, de classes de Wadge non auto-duales telle que

$$
\Gamma=\left\{\bigcup_{n \in \omega} A_{n} \cap U_{n}:\left(U_{n}\right) \boldsymbol{\Delta}_{1}^{0} \text {-partition, } A_{n} \in \Gamma_{n}\right\},
$$

ou bien $\Gamma$ est le successeur d'une classe non auto-duale $\Gamma^{\prime}$ telle que

$$
\Gamma=\left\{(A \cap N) \cup(B \backslash N): N \in \boldsymbol{\Delta}_{1}^{0}, A \in \Gamma^{\prime}, B \in \check{\Gamma}^{\prime}\right\} .
$$

Dans la première éventualité, comme $\Gamma_{n}$ est non auto-duale, on trouve $A_{n}$ dans $\Gamma_{n}\left\lceil\omega^{\omega} \times \omega^{\omega}\right.$ tel que $\Gamma_{A_{n}}=\Gamma_{n}$ et si $B_{n}$ est dans $\Gamma_{n}\left\lceil\omega^{\omega} \times \omega^{\omega}\right.$, il existe $\alpha_{n}$ dans $\omega^{\omega}$ tel que $B_{n}(\gamma, \beta) \Leftrightarrow A_{n}\left(\left\langle\alpha_{n}, \gamma\right\rangle, \beta\right)$ (ceci par le premier cas).

Soit

$$
\psi_{n}: \omega^{\omega} \rightarrow N_{(n)}, \quad \alpha \mapsto n \frown \alpha,
$$

et $B:=\bigcup_{n \in \omega}\left(\psi_{n} \times \mathrm{Id}\right)^{\prime \prime} A_{n}$. La fonction $\psi_{n}$ étant un homéomorphisme, $\left(\psi_{n} \times \mathrm{Id}\right)^{\prime \prime} A_{n} \in \Gamma_{n}\left\lceil N_{(n)} \times \omega^{\omega} \subseteq \Gamma\left\lceil N_{(n)} \times \omega^{\omega}\right.\right.$ et $B \in \boldsymbol{\Delta}_{1}^{0}-\mathrm{PU}(\Gamma)=\Gamma$, donc $\Gamma_{B} \subseteq \Gamma$.

Si l'inclusion est stricte, il existe $n$ tel que $\Gamma_{B} \psi \Gamma_{n}=\Gamma_{A_{n}}$; or on a $A_{n}=\left(\psi_{n} \times \mathrm{Id}\right)^{-1}(B)$, et $\Gamma_{A_{n}} \subseteq \Gamma_{B}$ par le lemme précédent, d'où contradiction. Donc $\Gamma_{B}=\Gamma$.

Si $A$ est $\operatorname{Pot}(\Gamma)$, on trouve $\sigma^{\prime}, \tau^{\prime}, F, H, \varphi, \psi, R$ comme au premier cas. On sait qu'on peut trouver une $\boldsymbol{\Delta}_{1}^{0}$-partition $\left(U_{n}\right)$ de $\omega^{\omega} \times \omega^{\omega}$ et $B_{n}$ dans $\Gamma_{n}\left\lceil\omega^{\omega} \times \omega^{\omega}\right.$ tels que l'on ait l'égalité $R=\bigcup_{n \in \omega} U_{n} \cap B_{n}$. La fonction $f:=h \circ(\varphi \times \psi) \circ$ Id convient, si on pose

$$
h: F \times H \rightarrow \omega^{\omega} \times \omega^{\omega}, \quad(\gamma, \beta) \mapsto\left(\psi_{n}\left(\left\langle\alpha_{n}, \gamma\right\rangle\right), \beta\right) \quad \text { si }(\gamma, \beta) \in U_{n},
$$

puisque si $C$ et $D$ sont des boréliens de $\omega^{\omega}$, on a

$$
h^{-1}(C \times D)=\bigcup_{n \in \omega} U_{n} \cap\left(\left\{\alpha \in \omega^{\omega}: \psi_{n}\left(\left\langle\alpha_{n}, \alpha\right\rangle\right) \in C\right\} \times D\right) \in \operatorname{Pot}\left(\Sigma_{1}^{0}\right) .
$$

Dans la seconde éventualité, on trouve $A_{0}$ dans $\Gamma^{\prime}\left\lceil\omega^{\omega} \times \omega^{\omega}\right.$, et $A_{1}$ dans $\check{\Gamma}^{\prime}\left[\omega^{\omega} \times \omega^{\omega}\right.$ tels que $\Gamma_{A_{0}}=\Gamma^{\prime}, \Gamma_{A_{1}}=\check{\Gamma}^{\prime}$; et si $C$ (resp. D) est dans $\Gamma^{\prime}$ (resp. $\Gamma)\left\lceil\omega^{\omega} \times \omega^{\omega}\right.$, on trouve $\alpha_{0}$ (resp. $\alpha_{1}$ ) dans $\omega^{\omega}$ tels que

$$
C(\gamma, \beta) \Leftrightarrow A_{0}\left(\left\langle\alpha_{0}, \gamma\right\rangle, \beta\right), \quad D(\gamma, \beta) \Leftrightarrow A_{1}\left(\left\langle\alpha_{1}, \gamma\right\rangle, \beta\right) .
$$


Si $\varphi_{0}$ est un homéomorphisme de $\omega^{\omega}$ sur $\omega^{\omega} \backslash N_{(0)}$, et si

$$
B:=\left(\varphi_{0} \times \mathrm{Id}\right)^{\prime \prime} A_{1} \cup\left(\psi_{0} \times \mathrm{Id}\right)^{\prime \prime} A_{0},
$$

$B$ est dans $\Gamma\left\lceil\omega^{\omega} \times \omega^{\omega}\right.$, donc $\Gamma_{B} \subseteq \Gamma$.

Comme $\Gamma$ est le successeur de $\Gamma^{\prime}$, si l'inclusion est stricte, on a $\Gamma_{B} \subseteq \Gamma^{\prime}$ ou $\Gamma_{B} \subseteq \check{\Gamma}^{\prime}$. Soit par exemple $\Gamma_{B} \subseteq \Gamma^{\prime} ; A_{1} \in \operatorname{Pot}\left(\check{\Gamma}^{\prime}\right)=\operatorname{Pot}\left(\Gamma_{A_{1}}\right)$, et comme $\Gamma^{\prime}$ est non auto-duale, $A_{1}$ n'est pas $\operatorname{Pot}\left(\Gamma^{\prime}\right)$ car $\Gamma_{A_{1}}=\check{\Gamma}^{\prime}$. Donc $A_{1}$ n'est pas $\operatorname{Pot}\left(\Gamma_{B}\right)$; or $A_{1}=\left(\varphi_{0} \times \mathrm{Id}\right)^{-1}(B)$, donc $\Gamma_{A_{1}} \subseteq \Gamma_{B}$, une contradiction. La dernière partie est analogue à celle de la première éventualité.

On cherche maintenant à adapter les résultats sur les classes de Wadge. Si $C$ (resp. $D)$ est borélien de $X$ (resp. $Y$ ), on a

$\langle C\rangle \subseteq\langle D\rangle \Leftrightarrow$ il existe $f$ continue, de $X$ dans $Y$, telle que $C=f^{-1}(D)$.

Une question analogue se pose pour les classes de Wadge potentielles : peut-on trouver une classe de fonctions $\mathcal{C}$ telle que si $B$ est borélien de $Z \times T$, on ait

$$
\Gamma_{A} \subseteq \Gamma_{B} \Leftrightarrow \exists f: X \times Y \rightarrow Z \times T \text { dans } \mathcal{C} \text { telle que } A=f^{-1}(B) .
$$

Comme on va le voir, la réponse est négative; la classe qui semblait le candidat "raisonnable", $C_{0}$ à cause du lemme précédent, ne fonctionne pas, et à un petit niveau (avec $A \in \boldsymbol{\Pi}_{1}^{0}$ et $B \in \check{D}_{2}\left(\boldsymbol{\Sigma}_{1}^{0}\right)$ ).

On notera $\leq_{P}$ le pré-ordre associé à $C_{0}$ :

$$
A \leq_{P} B \Leftrightarrow \text { il existe } f \text { dans } C_{0} \text { telle que } A=f^{-1}(B) .
$$

L'inégalité $A \leq_{P} B$ entraîne donc l'inclusion $\Gamma_{A} \subseteq \Gamma_{B}$, par le lemme 3.2. On montre maintenant un lemme bien plus fort que nécessaire pour introduire le contre-exemple évoqué ci-dessus, mais qui permettra de mieux comprendre ce qu'on cherche à faire dans les paragraphes suivants.

DÉfinition 3.4. Si $X$ est un espace polonais, on dira que $G, G_{\delta}$ de $X$, est presque-ouvert si $G$ est contenu dans l'intérieur de son adhérence.

Lemme 3.5. Soient $\left(C_{n}\right)$ (resp. $\left(D_{n}\right)$ ) des suites de presque-ouverts non vides de $X$ (resp. $Y$ ), $f_{n}: C_{n} \rightarrow D_{n}$ continues et ouvertes, $B$ la réunion $\bigcup_{n \in \omega \backslash\{0\}} \operatorname{Gr}\left(f_{n}\right)$, et $A$ un borélien de $X \times Y$ contenant $B$; si $\bar{B} \backslash A$ contient $\operatorname{Gr}\left(f_{0}\right)$, alors $A$ n'est pas $\operatorname{Pot}\left(\boldsymbol{\Pi}_{1}^{0}\right)$.

Démonstration. Sinon, soit $F$ (resp. $G$ ) un $G_{\delta}$ dense de $X$ (resp. $Y$ ) sur lequel les topologies (initiales et fournies par le fait que $A$ soit $\operatorname{Pot}\left(\boldsymbol{\Pi}_{1}^{0}\right)$ ) coïncident (on montre leur existence comme dans la preuve du théorème 3.3 ); on a $A \cap(F \times G) \in \boldsymbol{\Pi}_{1}^{0}\lceil F \times G$.

Montrons que $\operatorname{Gr}\left(f_{n}\right) \subseteq \overline{\operatorname{Gr}\left(f_{n}\right) \cap(F \times G)}$. Soit $U$ (resp. $V$ ) un ouvert de $X$ (resp. $Y$ ) tels que $(U \times V) \cap \operatorname{Gr}\left(f_{n}\right) \neq \emptyset$. Alors $D_{n} \cap V \cap G$ est un $G_{\delta}$ dense de $D_{n} \cap V$, donc $f_{n}^{-1}(V \cap G)$ est un $G_{\delta}$ dense de $f_{n}^{-1}(V)$. Donc $F \cap f_{n}^{-1}(V)$, puis $F \cap f_{n}^{-1}(V \cap G)$, sont des $G_{\delta}$ denses de $f_{n}^{-1}(V)$; 
ce dernier rencontre donc $U \cap f_{n}^{-1}(V)$ en au moins $\{x\}$; alors $\left(x, f_{n}(x)\right)$ est dans $(U \times V) \cap(F \times G) \cap \operatorname{Gr}\left(f_{n}\right) \neq \emptyset$.

$\operatorname{Gr}\left(f_{0}\right)$ est non vide, donc par ce qui précède on trouve $(x, y)$ dans $(F \times G) \cap \operatorname{Gr}\left(f_{0}\right)$, et on a $(x, y) \in(F \times G) \cap \bar{B} \backslash A$; il suffit donc de voir que $(F \times G) \cap \bar{B} \subseteq(F \times G) \cap \overline{B \cap(F \times G)}$. On applique alors le fait que $\operatorname{Gr}\left(f_{n}\right) \subseteq \overline{\operatorname{Gr}\left(f_{n}\right) \cap(F \times G)}$ pour avoir la contradiction cherchée.

Exemple 3.6. Soit $D_{0}:=\left\{(\alpha, \beta) \in \mathbf{2}^{\omega} \times \mathbf{2}^{\omega}: \exists ! p \in \omega, \alpha(p) \neq \beta(p)\right\}$. Alors $D_{0}$ n'est pas $\operatorname{Pot}\left(\boldsymbol{\Pi}_{1}^{0}\right)$.

En effet, on applique le lemme précédent à $X=Y=C_{n}=D_{n}=\mathbf{2}^{\omega}$, $f_{0}(\alpha)=\alpha, f_{n}(\alpha)(p)=\alpha(p) \Leftrightarrow p \neq n-1$ si $n>0$, et $A=B$.

ThÉORÈme 3.7. Il n'existe pas de classe de fonctions $\mathcal{C}$ telle que l'inclusion de $\Gamma_{A}$ dans $\Gamma_{B}$ soit équivalente à l'existence de $f$ dans $\mathcal{C}$ telle que $A=f^{-1}(B)$.

Démonstration. Raisonnons par l'absurde; si $B$ est $\operatorname{Pot}\left(\boldsymbol{\Sigma}_{1}^{0}\right)$ et $f$ dans $\mathcal{C}$, comme $\Gamma_{B} \subseteq \boldsymbol{\Sigma}_{1}^{0}, \Gamma_{f^{-1}(B)} \subseteq \boldsymbol{\Sigma}_{1}^{0}$, donc $f^{-1}(B)$ est $\operatorname{Pot}\left(\boldsymbol{\Sigma}_{1}^{0}\right)$. Donc si $\mathcal{C}$ existe, $\mathcal{C}$ est une sous-classe de $C_{0}$.

Comme on l'a vu avant le lemme 3.2, $\Gamma_{\Delta\left(2^{\omega}\right)}=\Pi_{1}^{0}$, et par 3.6, $\check{D}_{0}$ n'est pas $\operatorname{Pot}\left(\boldsymbol{\Sigma}_{1}^{0}\right)$, donc $\boldsymbol{\Pi}_{1}^{0} \subseteq \Gamma_{\check{D}_{0}}$ et $\Gamma_{\Delta\left(\mathbf{2}^{\omega}\right)} \subseteq \Gamma_{\check{D}_{0}}$. Il suffit donc de voir que $\Delta\left(\mathbf{2}^{\omega}\right) \mathbb{E}_{P} \check{D}_{0}$ pour avoir la contradiction cherchée.

Raisonnons par l'absurde : il existe $f$ dans $C_{0}$ telle que $\Delta\left(\mathbf{2}^{\omega}\right)=f^{-1}\left(\check{D}_{0}\right)$. Alors $f^{\prime \prime}\left(\Delta\left(\mathbf{2}^{\omega}\right)\right)$ est non dénombrable, sinon par la remarque $2.1, f^{\prime \prime}\left(\Delta\left(\mathbf{2}^{\omega}\right)\right)$ serait $\operatorname{Pot}\left(\boldsymbol{\Sigma}_{1}^{0}\right)$, et par suite $\Delta\left(\boldsymbol{2}^{\omega}\right)=f^{-1}\left(f^{\prime \prime}\left(\Delta\left(\mathbf{2}^{\omega}\right)\right)\right)$ aussi, ce qui est exclus.

On peut donc trouver une copie $P$ de $\mathbf{2}^{\omega}$ dans $\Delta\left(\mathbf{2}^{\omega}\right)$ sur laquelle $f$ est injective; $f^{\prime \prime} P$ est donc un borélien non dénombrable, et ses coupes sont dénombrables : si par exemple une de ses coupes verticales $C$ est non dénombrable, soit $\left(f^{\prime \prime} P\right)\left(\alpha_{0}\right),\left\{\alpha_{0}\right\} \times C$ est un rectangle borélien, donc est $\operatorname{Pot}\left(\boldsymbol{\Sigma}_{1}^{0}\right) ; f^{-1}\left(\left\{\alpha_{0}\right\} \times C\right)$ est alors aussi $\operatorname{Pot}\left(\boldsymbol{\Sigma}_{1}^{0}\right)$, non dénombrable car $\left\{\alpha_{0}\right\} \times C \subseteq f^{\prime \prime}\left(\mathbf{2}^{\omega} \times \mathbf{2}^{\omega}\right)$, et contenu dans $\Delta\left(\mathbf{2}^{\omega}\right)$, ce qui est contradictoire.

$f^{\prime \prime} P$ n'est donc pas réunion dénombrable de rectangles boréliens dont l'un des côtés soit un singleton, sinon les côtés seraient dénombrables comme les coupes, et $P$ aussi. Par le théorème 2.3, il existe un homéomorphisme $\psi$ de $\mathbf{2}^{\omega}$ sur un compact $L$, et une injection continue $g$ définie sur $L$, dont le graphe est contenu dans $f^{\prime \prime} P$.

Alors si $B:=D_{0} \cap\left(L \times g^{\prime \prime} L\right), B$ est borélien à coupes dénombrables de $L \times g^{\prime \prime} L$, donc est maigre relativement à $L \times g^{\prime \prime} L$. Donc, si l'on pose $E:=(\psi \times(g \circ \psi))^{-1}(B), E$ est maigre relativement à $\mathbf{2}^{\omega} \times \mathbf{2}^{\omega}$, et par la proposition 1.6, il existe $M$ homéomorphe à $\mathbf{2}^{\omega}$ tel que si $\alpha$ et $\beta$ sont distincts dans $M$, alors $(\alpha, \beta)$ n'est pas dans $E$. 
Soit $R:=\psi^{\prime \prime} M \times(g \circ \psi)^{\prime \prime} M$; alors $R \subseteq \check{D}_{0}$, sinon soit $(\alpha, \beta)$ dans $R \cap D_{0}$; on a alors $\alpha=\psi(\theta)$ et $\beta=g(\psi(\varepsilon))$, où $\theta, \varepsilon$ sont dans $M$, et $\theta \neq \varepsilon$, sinon $(\alpha, \beta) \in \operatorname{Gr}(g) \subseteq f^{\prime \prime} P \subseteq \check{D}_{0}$. Donc $(\theta, \varepsilon) \notin E$ et $(\alpha, \beta) \notin B$, une contradiction.

De plus, $\operatorname{Gr}\left(g\left\lceil\psi^{\prime \prime} M\right) \subseteq R \cap f^{\prime \prime} P\right.$, donc $R \cap f^{\prime \prime} P$ est non dénombrable, et $f^{-1}(R)$ est $\operatorname{Pot}\left(\boldsymbol{\Sigma}_{1}^{0}\right)$ comme $R$, est contenu dans $\Delta\left(\mathbf{2}^{\omega}\right)$, et est non dénombrable, ce qui est exclus.

4. Résultats de type "Hurewicz". Dans [Lo-SR], il est démontré le résultat suivant :

ThÉORÈme 4.1. Si $\xi$ est un ordinal dénombrable non nul, il existe un compact de dimension $0, P_{\xi}$, et un vrai $\Sigma_{\xi}^{0}$ de $P_{\xi}, A_{\xi}$, tels que si $A$ est un borélien de l'espace polonais $X$, on ait: $A$ n'est pas $\boldsymbol{\Pi}_{\xi}^{0}$ de $X$ si et seulement s'il existe une injection continue $f$ de $P_{\xi}$ dans $X$ telle que $A_{\xi}=f^{-1}(A)$.

En fait $P_{\xi}=\mathbf{2}^{\omega}$, sauf si $\xi=1$, auquel cas $P_{1}$ est constitué d'une suite convergente infinie et de sa limite. Ceci implique, avec $B=f^{\prime \prime} P_{\xi}$, que $A$ n'est pas $\boldsymbol{\Pi}_{\xi}^{0}$ si et seulement s'il existe un fermé $B$ de $X$ tel que $A \cap B$ soit un vrai $\Sigma_{\xi}^{0}$ de $B$. L'ensemble $A_{\xi}$ est dit "test d'Hurewicz".

Dans la suite, on cherchera à établir un analogue à ces résultats dans le cas où $\xi=1$. On y parviendra partiellement; dans cet esprit, voici la

DÉfinition 4.2. Si $\Gamma$ est une classe, on dira que $P_{1}(\Gamma)$ est vérifiée si, pour tout $A$ dans $\Gamma, A$ n'est pas $\operatorname{Pot}\left(\boldsymbol{\Pi}_{1}^{0}\right)$ si et seulement s'il existe un $\operatorname{Pot}\left(\boldsymbol{\Pi}_{1}^{0}\right), B$, et un $\operatorname{Pot}\left(\boldsymbol{\Sigma}_{1}^{0}\right), C$, tels que $B \cap C=B \cap A$ ne soit pas $\operatorname{Pot}\left(\boldsymbol{\Pi}_{1}^{0}\right)$.

En apparence, cette propriété n'explique pas ce que signifie “ $A$ n'est pas $\operatorname{Pot}\left(\boldsymbol{\Pi}_{1}^{0}\right)$ ". Mais elle ramène le problème au cas où $A$ est $\operatorname{Pot}\left(D_{2}\left(\boldsymbol{\Sigma}_{1}^{0}\right)\right)$, et on va voir que sous l'hypothèse " $A$ est $\operatorname{Pot}\left(F_{\sigma}\right)$ ", on sait caractériser quand $A$ n'est pas $\operatorname{Pot}\left(\boldsymbol{\Pi}_{1}^{0}\right)$. Mais il nous faut la

DÉfinition 4.3. Si $X$ et $Y$ sont des espaces topologiques, une partie $A$ de $X \times Y$ sera dite localement à projections ouvertes (ou l.p.o.) si pour tout ouvert $U$ de $X \times Y$, les projections de $A \cap U$ sont ouvertes.

Lemme 4.4. Soient $X$ et $Y$ des espaces polonais, $F$ et $G$ des $G_{\delta}$ denses de $X$ et $Y$, et $A$ un $G_{\delta}$ l.p.o. non vide de $X \times Y$; alors $A \cap(F \times G)$ est non vide.

Démonstration. Soient $\left(U_{n}\right)$ et $\left(V_{n}\right)$ des suites d'ouverts denses, de $X$ et $Y$, telles que $F=\bigcap_{n \in \omega} U_{n}, G=\bigcap_{n \in \omega} V_{n}$, et $\left(F_{n}\right)$ une suite de fermés de $X \times Y$ telle que $A=\bigcap_{n \in \omega} \check{F}_{n}$. On construit par récurrence sur $n$ des suites d'ouverts non vides $\left(O_{n}\right)$ et $\left(T_{n}\right)$ de $X$ et $Y$ vérifiant :

(i) $\delta\left(O_{n}\right), \delta\left(T_{n}\right)<2^{-n}$, 
(ii) $O_{n} \times T_{n} \subseteq\left(U_{n} \times V_{n}\right) \backslash F_{n}$,

(iii) $A \cap\left(O_{n} \times T_{n}\right) \neq \emptyset$,

(iv) $\bar{O}_{n+1} \subseteq O_{n}, \bar{T}_{n+1} \subseteq T_{n}$.

Admettons avoir construit ces objets; $\left(\bar{O}_{n}\right)$ et $\left(\bar{T}_{n}\right)$ sont des suites décroissantes de fermés non vides dont les diamètres tendent vers 0 , donc leurs intersections sont $\{x\}$ et $\{y\}$; mais $(x, y) \in O_{n} \times T_{n} \subseteq \check{F}_{n} \cap\left(U_{n} \times V_{n}\right)$, donc $(x, y) \in A \cap(F \times G) \neq \emptyset$.

Admettons avoir construit $\left(O_{p}\right)_{p<n}$ et $\left(T_{p}\right)_{p<n}$ vérifiant les conditions demandées; alors par (iii), $\Pi_{X}^{\prime \prime}\left(A \cap\left(O_{n-1} \times T_{n-1}\right)\right)$ est un ouvert non vide de $X$, donc rencontre $U_{n}$ : l'ensemble $A \cap\left[\left(O_{n-1} \cap U_{n}\right) \times T_{n-1}\right]$ est non vide, donc sa projection sur $Y$ est un ouvert non vide de $Y$, donc rencontre $V_{n}$. L'ensemble $A \cap\left[\left(O_{n-1} \cap U_{n}\right) \times\left(T_{n_{1}} \cap V_{n}\right)\right]$ est non vide, donc contient $\left(x_{n}, y_{n}\right)$; il reste à choisir deux ouverts $O_{n}$ et $T_{n}$ de diamètre au plus $2^{-n}$ vérifiant la double inclusion suivante :

$$
\left(x_{n}, y_{n}\right) \in O_{n} \times T_{n} \subseteq \overline{O_{n} \times T_{n}} \subseteq\left[\left(O_{n-1} \cap U_{n}\right) \times\left(T_{n-1} \cap V_{n}\right)\right] \backslash F_{n} .
$$

On remarquera que ce résultat est faux si on ne suppose pas que $A$ est un $G_{\delta}$. Désignons par $P_{\infty}$ l'ensemble des suites de 0 et de 1 comportant une infinité de termes égaux à 1 . Si maintenant $A$ désigne $\left(\mathbf{2}^{\omega} \times \mathbf{2}^{\omega}\right) \backslash\left(P_{\infty} \times P_{\infty}\right)$, $A$ est un $K_{\sigma}$ qui vérifie les autres conditions du lemme et ne rencontre pas $P_{\infty} \times P_{\infty} !$

ThÉorème 4.5. (a) Soit $A$ un $\operatorname{Pot}\left(F_{\sigma}\right)$ d'un produit d'espaces polonais $X \times Y$; alors $A$ n'est pas $\operatorname{Pot}\left(\boldsymbol{\Pi}_{1}^{0}\right)$ si et seulement s'il existe des espaces polonais de dimension $0, Z$ et $T$, une suite de fermés de $Z \times T,\left(F_{n}\right)$, et des fonctions injectives continues, $f$, de $Z$ dans $X$, et $g$, de $T$ dans $Y$, tels que si $C:=\bigcup_{n \in \omega} F_{n}$, on ait :

(i) $\bar{C} \backslash C \neq \emptyset$,

(ii) $C=(f \times g)^{-1}(A)$,

(iii) $\bar{C} \backslash C$ et $F_{n}$ sont l.p.o.

(b) Soit $A$ un $\operatorname{Pot}\left(\boldsymbol{\Delta}_{2}^{0}\right)$ d'un produit d'espaces polonais $X \times Y$; alors $A$ n'est pas $\operatorname{Pot}\left(\boldsymbol{\Pi}_{1}^{0}\right)$ si et seulement s'il existe des espaces polonais de dimension $0, Z$ et $T$, un $\boldsymbol{\Delta}_{2}^{0}$ de $Z \times T, C$, et des fonctions injectives continues, $f$, de $Z$ dans $X$, et $g$, de $T$ dans $Y$, tels que:

(i) $\bar{C} \backslash C \neq \emptyset$,

(ii) $C=(f \times g)^{-1}(A)$,

(iii) $\bar{C} \backslash C$ et $C$ sont l.p.o.

Démonstration. (a) Raisonnons par l'absurde : si $A$ est $\operatorname{Pot}\left(\boldsymbol{\Pi}_{1}^{0}\right)$, $C$ aussi et on trouve des $G_{\delta}$ denses $F$ et $G$ de $Z$ et $T$ tels que $C \cap(F \times G)$ soit dans $\boldsymbol{\Pi}_{1}^{0}\lceil F \times G$. 
On applique le lemme précédent à $Z, T, F, G$, et $\bar{C} \backslash C$, et on a $[(F \times G) \cap \bar{C}] \backslash C \neq \emptyset$. Il suffit alors de voir que ce dernier ensemble est égal à $[(F \times G) \cap \overline{C \cap(F \times G)}] \backslash C$ pour avoir la contradiction cherchée; et il suffit de voir que $F_{n} \subseteq \overline{F_{n} \cap(F \times G)}$.

Si $U$ et $V$ sont des ouverts de $Z$ et $T$ tels que $F_{n} \cap(U \times V)$ soit non vide, on applique le lemme précédent à $U, V, F \cap U, G \cap V$, et $F_{n} \cap(U \times V)$ pour voir que $F_{n} \cap[(F \cap U) \times(G \cap V)]$ est lui aussi non vide.

Inversement, on peut supposer que $X$ et $Y$ sont des fermés de $\omega^{\omega}$, et pour simplifier l'écriture qu'ils sont $\Delta_{1}^{1}$, ainsi que la suite $\left(G_{n}\right)$ de fermés pour $\Delta^{2}$ dont $A$ est la réunion (on applique la remarque $1.5(\mathrm{~b})$ ).

Comme $\Delta \subseteq \Sigma$, et par une double application du théorème de séparation, on voit que $\bar{A}^{\Sigma^{2}}=\bar{A}^{\Delta^{2}}$; par suite, puisque $\Delta$ est polonaise, $\bar{A}^{\Sigma^{2}} \backslash A$ est un $\Sigma_{1}^{1}$ non vide, ainsi que $\left(\bar{A}^{\Sigma^{2}} \backslash A\right) \cap \Omega_{\omega^{\omega} \times \omega^{\omega}}$; et puisque $\Omega_{\omega^{\omega} \times \omega^{\omega}} \subseteq \Omega_{\omega^{\omega}}^{2}$, $\left(\bar{A}^{\Sigma^{2}} \backslash A\right) \cap \Omega_{\omega^{\omega}}^{2}$ est lui aussi non vide; posons $Z:=\left(X \cap \Omega_{\omega^{\omega}}, \Sigma\left\lceil X \cap \Omega_{\omega^{\omega}}\right)\right.$, $T:=\left(Y \cap \Omega_{\omega^{\omega}}, \Sigma\left\lceil Y \cap \Omega_{\omega^{\omega}}\right), F_{n}:=G_{n} \cap(Z \times T)\right.$, et prenons pour $f$ et $g$ les applications identiques.

Alors $\left(\bar{A}^{\Sigma^{2}} \backslash A\right) \cap \Omega_{\omega^{\omega}}^{2}=\overline{A \cap(Z \times T)}^{\Sigma^{2}} \cap(Z \times T) \backslash(A \cap(Z \times T))$, donc ces objets conviennent : $\bar{C} \backslash C$ et $F_{n}$ sont $\Sigma_{1}^{1}$ et un ouvert de $Z \times T$ est réunion de rectangles $\Sigma_{1}^{1}$ (les projections des traces de ces rectangles seront donc $\left.\Sigma_{1}^{1}\right)$.

(b) Si $A$ n'est pas $\operatorname{Pot}\left(\boldsymbol{\Pi}_{1}^{0}\right)$, on raisonne comme dans (a), à ceci près qu'on pose $C:=A \cap(Z \times T)$.

La réciproque est analogue à celle de $(\mathrm{a})$, à ceci près que pour montrer l'égalité entre $[(F \times G) \cap \bar{C}] \backslash C$ et $[(F \times G) \cap \overline{C \cap(F \times G)}] \backslash C$, il suffit de voir que $C \subseteq \overline{C \cap(F \times G)}$; si $U$ et $V$ sont des ouverts de $Z$ et $T$ tels que $C \cap(U \times V)$ soit non vide, on applique le lemme 4.4 à $U, V, F \cap U, G \cap V$, et $C \cap(U \times V)$.

On introduit maintenant une propriété, qui est du type Hurewicz au sens de l'introduction; à ceci près que pour comparer la complexité, on n'a pas de réduction sur tout l'espace de départ, mais seulement sur un fermé.

DÉfinition 4.6. Si $\Gamma$ est une classe, on dira que $P_{2}(\Gamma)$ est vérifiée si pour tout $A$ dans $\Gamma\left\lceil X \times Y, A\right.$ n'est pas $\operatorname{Pot}\left(\boldsymbol{\Pi}_{1}^{0}\right)$ si et seulement s'il existe des espaces polonais de dimension $0, Z$ et $T$, une $D_{2}\left(\Sigma_{1}^{0}\right)$ de $Z \times T$, $D$, et des fonctions injectives continues, $f$, de $Z$ dans $X$, et $g$, de $T$ dans $Y$, tels que :

(i) $\bar{D} \backslash D \neq \emptyset$,

(ii) $\bar{D} \cap(f \times g)^{-1}(A)=D$,

(iii) $D$ et $\bar{D} \backslash D$ sont l.p.o.

Proposition 4.7. $P_{1}(\Gamma)$ équivaut à $P_{2}(\Gamma)$. 
Démonstration. Supposons $P_{1}(\Gamma)$; si $A \in \Gamma \backslash \operatorname{Pot}\left(\boldsymbol{\Pi}_{1}^{0}\right)$, soient $B$ et $C$ fournis par $P_{1}(\Gamma), \sigma$ et $\tau$ rendant $B$ fermé. Alors comme dans la preuve du théorème 4.5 (b) on trouve $Z, T$, et $D$ comme indiqué et des injections continues $F$, de $Z$ dans $(X, \sigma)$, et $G$, de $T$ dans $(Y, \tau)$, tels que l'on ait l'égalité $D=(F \times G)^{-1}(A) \cap(F \times G)^{-1}(B)$ (ceci parce que $B \cap C$ est $\left.\operatorname{Pot}\left(D_{2}\left(\Sigma_{1}^{0}\right)\right)\right)$. D'où $\bar{D} \cap(F \times G)^{-1}(A)=D$, et il ne reste qu'à revenir aux topologies initiales pour obtenir $f$ et $g$.

Inversement, si $A \in \operatorname{Pot}\left(\boldsymbol{\Pi}_{1}^{0}\right), D$ aussi, ce qui est exclus, comme dans la preuve du théorème $4.5(\mathrm{~b})$.

Supposons maintenant $P_{2}(\Gamma) ; \operatorname{Pot}\left(\boldsymbol{\Pi}_{1}^{0}\right)$ étant stable par intersection finie, $A$ n'est pas $\operatorname{Pot}\left(\boldsymbol{\Pi}_{1}^{0}\right)$ si $B$ et $C$ existent.

Inversement, si $A$ n'est pas $\operatorname{Pot}\left(\boldsymbol{\Pi}_{1}^{0}\right)$, soient $Z, T, D, f$, et $g$ fournis par $P_{2}(\Gamma) ; D=U \cap \bar{D}$, où $U$ est ouvert de $Z \times T$, donc $B:=(f \times g)^{\prime \prime} \bar{D}$ et $C:=(f \times g)^{\prime \prime} U$ répondent au problème, par injectivité de $f$ et $g$ : on a $B \cap A=(f \times g)^{\prime \prime} D=C \cap B$.

Cette proposition permet de comprendre pourquoi, indirectement, la propriété $P_{1}$ permet de mieux connaître les boréliens non potentiellement fermés. On établit maintenant cette propriété pour certaines familles de boréliens.

Proposition 4.8. La propriété $P_{1}$ est vérifiée par chacune des classes suivantes:

(i) les ensembles potentiellement $\boldsymbol{\Delta}_{2}^{0}$,

(ii) les boréliens à coupes verticales co-dénombrables,

(iii) les relations d'équivalence boréliennes.

Avant de démontrer cette proposition, on donne la définition suivante :

DÉfinition 4.9. Si $\Gamma$ est une classe, on dira que $P_{3}(\Gamma)$ est vérifiée si pour tout $A$ dans $\Gamma, A$ n'est pas $\operatorname{Pot}\left(\boldsymbol{\Sigma}_{1}^{0}\right)$ si et seulement s'il existe $B$ dans $\operatorname{Pot}\left(\boldsymbol{\Pi}_{1}^{0}\right)$ tel que $A \cap B$ soit $\operatorname{Pot}\left(\boldsymbol{\Pi}_{1}^{0}\right)$, et tel que pour tout $C \operatorname{dans} \operatorname{Pot}\left(\boldsymbol{\Sigma}_{1}^{0}\right)$, $A \cap B \neq C \cap B$.

Il est clair, en raison des formules $B \backslash A=B \backslash(A \cap B)$ et $B \cap A=B \backslash(B \backslash A)$ que $P_{1}(\Gamma)$ équivaut à $P_{3}(\Gamma)$ si $\Gamma$ est auto-duale; mais cette dernière est plus maniable quand il est question de réunions dénombrables.

Démonstration de la proposition 4.8. On a démontré le "si" dans le cas général; on montre donc la réciproque dans chacun des trois cas.

(i) On montre la chose plus précise suivante : si $\Gamma$ est un contre-exemple minimal (pour l'ordre de Wadge) à $P_{3}(\operatorname{Pot}(\Gamma)), \Gamma$ est non auto-duale et est de niveau au moins deux.

Si $\Gamma$ est auto-duale, traitons le premier cas de l'alternative évoquée dans la preuve du théorème 3.3 (l'autre cas étant plus simple) : on trouve une 
partition $\left(U_{n}\right)$ de $X \times Y$ en $\operatorname{Pot}\left(\boldsymbol{\Delta}_{1}^{0}\right)$ et $A_{n}$ dans $\operatorname{Pot}\left(\Gamma_{n}\right)$, où $\Gamma_{n} \boldsymbol{\psi} \Gamma$, avec $A \cap U_{n}=A_{n} \cap U_{n} ; A=\bigcup_{n \in \omega} A_{n} \cap U_{n}$, donc il existe $n$ tel que $A_{n} \cap U_{n}$ ne soit pas $\operatorname{Pot}\left(\Sigma_{1}^{0}\right)$. Puisque $A_{n} \cap U_{n}$ est $\operatorname{Pot}\left(\Gamma_{n}\right)$, par minimalité de $\Gamma$, il existe donc $B^{\prime}$ dans $\operatorname{Pot}\left(\boldsymbol{\Pi}_{1}^{0}\right)$ tel que $A_{n} \cap U_{n} \cap B^{\prime}$ soit $\operatorname{Pot}\left(\boldsymbol{\Pi}_{1}^{0}\right)$ et pour tout $C$ dans $\operatorname{Pot}\left(\Sigma_{1}^{0}\right), A_{n} \cap U_{n} \cap B^{\prime} \neq B^{\prime} \cap C$. Il reste à poser $B:=B^{\prime} \cap U_{n}$.

Si $\Gamma$ est non auto-duale et de niveau au plus un, $\Gamma$ est de niveau un car $\Gamma$ contient les fermés, donc est de la forme $S D_{\eta}\left(\Delta, \Gamma_{*}\right): A=E \cup F$, où

$E=\bigcup_{\xi<\eta, n \in \omega} A_{\xi, n} \cap V_{\xi, n} \cap^{c}\left(\bigcup_{\theta<\xi, p \in \omega} V_{\theta, p}\right)$ et $F=A_{*} \cap^{c}\left(\bigcup_{\xi<\eta, n \in \omega} V_{\xi, n}\right)$,

$A_{\xi, n}$ étant $\operatorname{Pot}(\Delta),\left(V_{\xi, n}\right)_{n \in \omega}$ étant une suite $\operatorname{de} \operatorname{Pot}\left(\Sigma_{1}^{0}\right)$ deux à deux disjoints, et $A_{*}$ étant $\operatorname{Pot}\left(\Gamma_{*}\right)$, où $\check{\Gamma}_{*} \neq \Gamma_{*} \subseteq \Delta$.

Premier cas : E n'est pas $\operatorname{Pot}\left(\boldsymbol{\Sigma}_{1}^{0}\right)$. Soient $\left(V_{\xi, n, p}\right)_{p \in \omega}$ une partition de $V_{\xi, n}$ en ensembles $\operatorname{Pot}\left(\boldsymbol{\Delta}_{1}^{0}\right)$,

$$
\begin{aligned}
V_{n, p}^{\xi} & :=V_{\xi, n, p} \cap\left[\bigcup_{\theta<\xi, q \in \omega} A_{\theta, q} \cap V_{\theta, q} \cap^{c}\left(\bigcup_{\varrho<\theta, r \in \omega} V_{\varrho, r}\right)\right], \quad \text { et } \\
D_{n, p}^{\xi} & :=\left[A_{\xi, n} \cap V_{\xi, n, p} \cap^{c}\left(\bigcup_{\theta<\xi, q \in \omega} V_{\theta, q}\right)\right] \cup V_{n, p}^{\xi} .
\end{aligned}
$$

Alors $E=\bigcup_{\xi<\eta ; n, p \in \omega} D_{n, p}^{\xi}$, donc on trouve $\xi$ minimal tel qu'il existe $n$ et $p$ tels que $D_{n, p}^{\xi}$ ne soit pas $\operatorname{Pot}\left(\boldsymbol{\Sigma}_{1}^{0}\right)$. Comme $V_{n, p}^{\xi}=V_{\xi, n, p} \cap \bigcup_{\theta<\xi ; q, r \in \omega} D_{q, r}^{\theta}$, $V_{n, p}^{\xi}$ est $\operatorname{Pot}\left(\boldsymbol{\Sigma}_{1}^{0}\right)$ par la minimalité de $\xi$; or

$$
\Delta=\left\{\begin{array}{l}
\Gamma_{0} \cup \check{\Gamma}_{0}, \\
\bigcup_{n \in \omega} \Gamma_{n},
\end{array}\right.
$$

où $\left(\Gamma_{n}\right)$ est une suite strictement croissante de classes de Wadge de niveau différent de un, donc sauf si

(1) " $\Gamma_{0}=\{\emptyset\}$ et $\Delta=\Gamma_{0} \cup \check{\Gamma}_{0} "$,

il existe $\Gamma^{\prime} \psi \Gamma$ telle que $D_{n, p}^{\xi}$ soit $\operatorname{Pot}\left(\Gamma^{\prime}\right)$.

Mais si on a (1), $D_{n, p}^{\xi}=G \cup V_{n, p}^{\xi}$, où $G:=D_{n, p}^{\xi} \backslash V_{n, p}^{\xi}$ est $\operatorname{Pot}\left(\boldsymbol{\Pi}_{1}^{0}\right)$; alors si on pose $B^{\prime}:=\check{V}_{n, p}^{\xi}, B^{\prime}$ est $\operatorname{Pot}\left(\boldsymbol{\Pi}_{1}^{0}\right), D_{n, p}^{\xi} \cap B^{\prime}=G$ aussi, et si $C$ est $\operatorname{Pot}\left(\boldsymbol{\Sigma}_{1}^{0}\right)$ et $C \cap B^{\prime}=B^{\prime} \cap D_{n, p}^{\xi}, D_{n, p}^{\xi}=\left(C \cap B^{\prime}\right) \cup V_{n, p}^{\xi}=C \cup V_{n, p}^{\xi}$ est $\operatorname{Pot}\left(\Sigma_{1}^{0}\right)$; par conséquent, $B^{\prime}$ vérifie les conclusions de la propriété $P_{3}$ avec $A:=D_{n, p}^{\xi}$.

Dans l'autre cas, par minimalité de $\Gamma$, on trouve aussi un $B^{\prime}$ vérifiant ces conclusions. Il reste à poser $B:=B^{\prime} \cap V_{\xi, n, p}$, puisque $A \cap B=B^{\prime} \cap D_{n, p}^{\xi}$.

Second cas : $E$ est $\operatorname{Pot}\left(\boldsymbol{\Sigma}_{1}^{0}\right)$. Dans ce cas $F$ n'est pas $\operatorname{Pot}\left(\boldsymbol{\Sigma}_{1}^{0}\right)$, et sauf si on a (1), on trouve $\Gamma^{\prime} \boldsymbol{\psi} \Gamma$ de niveau au moins deux telle que $F$ soit $\operatorname{Pot}\left(\Gamma^{\prime}\right)$, donc telle que $A$ soit $\operatorname{Pot}\left(\Gamma^{\prime}\right)$, et l'hypothèse de minimalité s'applique. Si on a (1), $F$ est $\operatorname{Pot}\left(\boldsymbol{\Pi}_{1}^{0}\right)$, ce qu'on a traité au premier cas. 
Si maintenant $A$ est $\operatorname{Pot}\left(\boldsymbol{\Delta}_{2}^{0}\right)$, il existe un ordinal dénombrable $\eta>1$ tel que $\check{A}$ soit $\operatorname{Pot}\left(D_{\eta}\left(\boldsymbol{\Sigma}_{1}^{0}\right)\right)$, par le théorème de Hausdorff. Comme $\Gamma$ est de niveau au moins deux, $D_{\eta}\left(\boldsymbol{\Sigma}_{1}^{0}\right) \boldsymbol{\psi} \boldsymbol{\Delta}_{2}^{0} \subseteq \Gamma$. Par ce qui précède, il existe $B$ dans $\operatorname{Pot}\left(\boldsymbol{\Pi}_{1}^{0}\right)$ tel que $B \backslash A$ soit $\operatorname{Pot}\left(\boldsymbol{\Pi}_{1}^{0}\right)$ et pour tout $H \operatorname{dans} \operatorname{Pot}\left(\boldsymbol{\Sigma}_{1}^{0}\right)$, $B \backslash A \neq B \cap H$. Alors $C:=A \cup B$ répond à la question.

(ii) Soit $A$ un borélien à coupes verticales co-dénombrables; on montre bien plus que la propriété $P_{1}$ :

(2) Il existe des copies $P$ et $Q$ de $\mathbf{2}^{\omega}$, et un homéomorphisme $\phi$ de $P$ dans $Q$ tels que $\operatorname{Gr}(\phi)=(P \times Q) \backslash A$.

Comme dans la démonstration du théorème $2.3, \check{A}=\bigcup_{n \in \omega} \operatorname{Gr}\left(f_{n}\right)$, où les fonctions $f_{n}$ sont boréliennes et définies sur des boréliens $B_{n}$ et on trouve un homéomorphisme $\phi_{0}$ de $2^{\omega}$ sur $R \subseteq B_{0}$, avec $f_{0}\lceil R$ injective continue. Posons

$$
E(\alpha, \beta) \Leftrightarrow \text { il existe } n>0 \text { tel que } \phi_{0}(\alpha) \in B_{n} \text { et } f_{0}\left(\phi_{0}(\beta)\right)=f_{n}\left(\phi_{0}(\alpha)\right) \text {. }
$$

Alors $E$ est borélien à coupes verticales dénombrables de $\mathbf{2}^{\omega} \times \mathbf{2}^{\omega}$, donc est maigre relativement à $\mathbf{2}^{\omega} \times \mathbf{2}^{\omega}$ et par la proposition 1.6 il existe dans $\mathbf{2}^{\omega}$ une copie $L$ de $\mathbf{2}^{\omega}$ telle que si $\alpha$ et $\beta$ sont distincts dans $L,(\alpha, \beta)$ n'est pas dans $E$.

Si $\alpha$ et $\beta$ sont distincts dans $\phi_{0}^{\prime \prime} L$, alors pour tout $n>0, \beta \notin B_{n}$ ou $f_{0}(\alpha) \neq f_{n}(\beta)$. Il reste à poser $P:=\phi_{0}^{\prime \prime} L, Q:=\left(f_{0} \circ \phi_{0}\right)^{\prime \prime} L, \phi:=f_{0}\lceil P$.

L'ensemble $(P \times Q) \backslash \operatorname{Gr}(\phi)$ n'est pas $\operatorname{Pot}\left(\boldsymbol{\Pi}_{1}^{0}\right)$, sinon $\operatorname{Gr}(\phi)$ serait $\operatorname{Pot}\left(\boldsymbol{\Sigma}_{1}^{0}\right)$ ainsi que $\Delta\left(\mathbf{2}^{\omega}\right)=(\operatorname{Id} \times \phi)^{-1}(\operatorname{Gr}(\phi))$. Les ensembles $B:=P \times Q$ et $C:=\operatorname{Gr}(\phi)$ répondent donc au problème.

(iii) Par le théorème de Harrington, Kechris, et Louveau (cf. [HKL]), on trouve une injection continue $f$ de $2^{\omega}$ dans $X$ telle que $E_{0}=(f \times f)^{-1}(A)$ $\left(E_{0}\right.$ étant l'ensemble des couples de suites infinies de 0 et de 1 égales à partir d'un certain rang).

Il suffit donc de regarder le cas où $A=E_{0}$. Posons, si $\alpha \in \mathbf{2}^{\omega}$,

$$
g_{0}(\alpha)(p)=1-\alpha(p),
$$

et

$$
g_{n}(\alpha)(p)=\alpha(p) \Leftrightarrow p>n-1 \quad \text { si } n>0 ;
$$

la suite $\left(g_{n}\right)$ est une suite d'homéomorphismes, et converge simplement vers $g$. Posons également $B:=\bigcup_{n \in \omega} \operatorname{Gr}\left(g_{n}\right), C:=\breve{G r}\left(g_{0}\right)$; par ce qui précède $B$ est borélien à coupes compactes, donc est $\operatorname{Pot}\left(\boldsymbol{\Pi}_{1}^{0}\right) ; C$ est ouvert, et on a la double égalité $B \cap A=C \cap B=\bigcup_{n \in \omega \backslash\{0\}} \operatorname{Gr}\left(g_{n}\right)$. Ce dernier ensemble n'est pas $\operatorname{Pot}\left(\boldsymbol{\Pi}_{1}^{0}\right)$, à cause du lemme 3.5 appliqué à $X=Y=C_{n}=D_{n}=\mathbf{2}^{\omega}$, $\left(g_{n}\right)$, et $A=B$. 
On donne maintenant un résultat qui est une condition suffisante pour obtenir la conclusion du (2) de la preuve précédente, mais sans borne sur la classe de Wadge potentielle de $A$ (par la remarque 1.5(a), un borélien à coupes verticales co-dénombrables est $\left.\operatorname{Pot}\left(\boldsymbol{\Pi}_{2}^{0}\right)\right)$.

Ce résultat se rattache aussi au théorème 2.3, qui implique que si $A$ n'est pas $\operatorname{Pot}\left(\boldsymbol{\Sigma}_{1}^{0}\right), A$ contient un graphe de fonction injective continue définie sur une copie de $\mathbf{2}^{\omega}$ (la réciproque étant fausse : prendre $A=\mathbf{2}^{\omega} \times \mathbf{2}^{\omega}$ !). Cependant, la réciproque est vraie si la trace de $A$ sur un rectangle parfait est un tel graphe, comme on l'a vu dans la preuve du (ii) de la proposition 4.8.

THÉORÈme 4.10. Soient $X$ et $Y$ des espaces polonais et $A$ un borélien à coupes horizontales maigres de $X \times Y$ tel que $\Pi_{X}^{\prime \prime} A$ soit non maigre; alors $i l$ existe une copie $P($ resp. $Q)$ de $\mathbf{2}^{\omega}$ dans $X($ resp. $Y)$, et un homéomorphisme $\phi$ de $P$ sur $Q$ tels que $\operatorname{Gr}(\phi)=(P \times Q) \cap A$.

Rappelons un lemme démontré dans $[\mathrm{Ke}]$ :

Lemme 4.11. Si $X$ et $Y$ sont des espaces polonais et $A$ est borélien à coupes horizontales maigres de $X \times Y, A$ est contenu dans une réunion dénombrable de boréliens à coupes fermées rares de $X \times Y$.

LEMme 4.12. Soient $G$ et $Y$ des espaces polonais, $f$ une fonction continue "meager-to-one" de $G$ dans $Y, O$ un ouvert à coupes horizontales denses de $G \times Y, \varepsilon>0$, et pour $i=0,1, M_{i}$ (resp. $\left.N_{i}\right)$ des ouverts non vides de $G$ (resp. $Y$ ) tels que $M_{i} \subseteq f^{-1}\left(N_{i}\right)$. Alors il existe des ouverts non vides $M_{i}^{\prime}($ de $G)$ et $N_{i}^{\prime}($ de $Y)$ tels que:

(1) $\overline{M_{i}^{\prime} \times N_{i}^{\prime}} \subseteq M_{i} \times N_{i}$,

(2) $\delta\left(M_{i}^{\prime} \times N_{i}^{\prime}\right)<\varepsilon$

(3) $N_{0}^{\prime} \cap N_{1}^{\prime}=\emptyset$,

(4) $M_{i}^{\prime} \times N_{1-i}^{\prime} \subseteq O$,

(5) $M_{i}^{\prime} \subseteq f^{-1}\left(N_{i}^{\prime}\right)$.

Démonstration. $f$ est "meager-to-one", donc on peut trouver $y_{i}$ dans $f^{\prime \prime} M_{i}, y_{0} \neq y_{1}$, et des ouverts $O_{i}$ de $Y$ avec $y_{i} \in O_{i} \subseteq \bar{O}_{i} \subseteq N_{i}$ et $O_{0} \cap O_{1}=\emptyset$.

Posons $R_{0}:=\left(M_{0} \cap f^{-1}\left(O_{0}\right)\right) \times \Pi_{Y}^{\prime \prime}\left[\left(M_{1} \times O_{1}\right) \cap \operatorname{Gr}(f)\right]$; remarquons que $R_{0}$ est non vide : en effet, si $x_{0}$ est un antécédent de $y_{0}$ dans $M_{0},\left(x_{0}, y_{1}\right)$ est dans $R_{0}$. On trouve alors $(x, y)$ dans $R_{0} \cap O$ : en effet, $R_{0} \backslash O$ est fermé de $R_{0}$ à coupes rares relativement à $M_{0} \cap f^{-1}\left(O_{0}\right)$ (qui est ouvert de $G$ ), donc est fermé rare de $R_{0}$. Soient $L_{1}$ et $N_{1}^{\prime}$ des ouverts de diamètre au plus $\varepsilon$ tels que $(x, y) \in L_{1} \times N_{1}^{\prime} \subseteq \bar{L}_{1} \times N_{1}^{\prime} \subseteq\left[\left(M_{0} \cap f^{-1}\left(O_{0}\right)\right) \times O_{1}\right] \cap O$.

Alors posons $R_{1}:=\left(M_{1} \cap f^{-1}\left(N_{1}^{\prime}\right)\right) \times \Pi_{Y}^{\prime \prime}\left[\left(L_{1} \times O_{0}\right) \cap \operatorname{Gr}(f)\right]$; là encore, $R_{1}$ est non vide : en effet, si $x_{1}$ est un antécédent de $y$ dans $M_{1},\left(x_{1}, f(x)\right)$ est dans $R_{1}$. On trouve $\left(x^{\prime}, y^{\prime}\right)$ dans $R_{1} \cap O$, comme avant, et aussi des 
ouverts $M_{1}^{\prime}, N_{0}^{\prime}$, de diamètre au plus $\varepsilon$, tels que l'on ait

$$
\left(x^{\prime}, y^{\prime}\right) \in M_{1}^{\prime} \times N_{0}^{\prime} \subseteq \bar{M}_{1}^{\prime} \times N_{0}^{\prime} \subseteq\left[\left(M_{1} \cap f^{-1}\left(N_{1}^{\prime}\right)\right) \times O_{0}\right] \cap O .
$$

Il reste à poser $M_{0}^{\prime}:=L_{1} \cap f^{-1}\left(N_{0}^{\prime}\right)$.

Démonstration du théorème 4.10. Soit $N$ un ouvert non vide de $X$ sur lequel l'analytique $\Pi_{X}^{\prime \prime} A$ est co-maigre. On peut alors appliquer le lemme 4.11 à $N, Y$, et $A \cap(N \times Y)$, ce qui fournit une suite croissante $\left(F_{n}\right)$ de boréliens à coupes fermées rares de $N \times Y$, dont la réunion contient $A \cap(N \times Y)$. Par le rappel 1.1(b) on trouve une topologie polonaise $\tau$ sur $Y$ affinant la topologie initiale de sorte que $F_{n}$ soit fermé de $N \times(Y, \tau)$.

Par le théorème de von Neumann, on trouve une fonction $f$ Bairemesurable uniformisant $A \cap(N \times Y)$ sur $N \cap \Pi_{X}^{\prime \prime} A$; soit alors $G$ un $G_{\delta}$ dense de $N$, contenu dans $N \cap \Pi_{X}^{\prime \prime} A$, sur lequel $f$ est continue ( $G$ existe car $N \cap \Pi_{X}^{\prime \prime} A$ est co-maigre dans $\left.N\right)$.

On construit des ouverts non vides $\left(V_{s}\right)_{s \in 2<\omega} \operatorname{de} G$ et $\left(W_{s}\right)_{s \in 2<\omega} \operatorname{de}(Y, \tau)$ vérifiant, si $U_{s}:=V_{s} \times W_{s}$,

(i) $\bar{U}_{s \frown i} \subseteq U_{s}$,

(ii) $\delta\left(U_{s}\right)<|s|^{-1}$ si $s \neq \emptyset$,

(iii) $W_{s \frown 0} \cap W_{s \frown 1}=\emptyset$,

(iv) $\forall s \neq t \in 2^{n+1}, V_{s} \times W_{t} \subseteq \check{F}_{n}$,

(v) $V_{s} \subseteq f^{-1}\left(W_{s}\right)$.

Si on a construit ces objets, posons

$$
\left\{\left(x_{\alpha}, y_{\alpha}\right)\right\}:=\bigcap_{n \in \omega} U_{\alpha\lceil n}=\bigcap_{n \in \omega} \bar{U}_{\alpha\lceil n} .
$$

Alors $\left\{\left(x_{\alpha}, y_{\alpha}\right): \alpha \in \mathbf{2}^{\omega}\right\}$ est une copie de $\mathbf{2}^{\omega}$ qui est le graphe d'une injection partielle $\phi$ (ceci résulte de (iii) et (v), qui assure la disjonction de $V_{s \frown 0}$ et $V_{s \frown 1}$ si $s$ est dans $\left.2^{<\omega}\right)$. Les projections de ce graphe définissent les copies $P$ et $Q$ de $\mathbf{2}^{\omega}$ annoncées. Par (v), $\phi$ est la restriction de $f$ à $P$, qui est contenu dans $G$, donc $\phi$ est un homéomorphisme de $P$ sur $Q$. L'inclusion du graphe de $\phi$ dans $(P \times Q) \cap A$ est alors claire. Inversement, si $\left(x_{\alpha}, y_{\beta}\right)$ est dans $(P \times Q) \backslash \operatorname{Gr}(\phi), \alpha \neq \beta$, donc par (iv) on trouve $n>0$ tel que $\alpha\left\lceil n \neq \beta\left\lceil n\right.\right.$; par conséquent, $\left(x_{\alpha}, y_{\beta}\right)$ n'est pas dans $F_{m}$ si $n \leq m+1$ et $\left(x_{\alpha}, y_{\beta}\right)$ n'est pas dans $A$.

Montrons donc que la construction est possible. On pose $V_{\emptyset}:=G$ et $W_{\emptyset}:=Y$. Admettons avoir construit $U_{s}$ pour $s$ dans $2^{\leq n}$ vérifiant les conditions (i) $-(\mathrm{v})$.

On va appliquer plusieurs fois le lemme 4.12, à $f$ et à des ouverts de $G$ et $(Y, \tau)$, ce qui est licite puisque $f$ est "meager-to-one" sur $N$, donc sur $G$ qui est co-maigre dans $N$. 
On commence à appliquer ce lemme 4.12 à $\varepsilon=(n+1)^{-1}$ et aux ouverts $O=(G \times Y) \backslash F_{n}, M_{0}=M_{1}=V_{s}$ et $N_{0}=N_{1}=W_{s}$, de sorte qu'on a assuré (i)-(iii) et (v), ainsi que (iv) pour les couples de la forme $(s \frown i, s \frown(1-i))$, avec $s$ dans $2^{n}$ et $i$ dans 2 (on obtient ainsi $\widetilde{V}_{s \frown 0}, \widetilde{V}_{s \frown 1}, \widetilde{W}_{s \frown 0}, \widetilde{W}_{s \frown 1}$ ). Si jamais, pour $u$ et $v$ distincts dans $2^{n+1}, \widetilde{V}_{u} \times \widetilde{W}_{v} \cap F_{n}$ est non vide, on diminue à l'aide du lemme 4.12 appliqué à $M_{0}=\widetilde{V}_{u}, M_{1}=\widetilde{V}_{v}, N_{0}=\widetilde{W}_{u}$, $N_{1}=\widetilde{W}_{v}, \widetilde{V}_{u}$ et $\widetilde{W}_{v}$ de manière à éviter $F_{n}$ tout en conservant $(\mathrm{v})$. On réalise ainsi (iv) au bout d'un nombre fini de changements éventuels (majoré par $\left.\left(2^{n+1}\right)^{2}\right)$.

Par le (i) de la proposition 4.8, on a $P_{1}\left(\operatorname{Pot}\left(\boldsymbol{\Delta}_{2}^{0}\right)\right)$, donc a fortiori $P_{1}\left(\operatorname{Pot}\left(\check{D}_{2}\left(\boldsymbol{\Sigma}_{1}^{0}\right)\right)\right)$; on va donner une nouvelle preuve de ceci mais sous une forme beaucoup plus forte, du type du théorème rappelé au début du paragraphe.

Comme il résulte de la preuve du (ii) de la proposition 4.8 , si $D$ est à coupes dénombrables et est non $\operatorname{Pot}\left(\boldsymbol{\Sigma}_{1}^{0}\right)$, on trouve des injections continues $\phi$ et $\psi$ telles que $\Delta\left(\mathbf{2}^{\omega}\right)=(\phi \times \psi)^{-1}(D)$, donc en particulier $\Delta\left(\mathbf{2}^{\omega}\right) \leq_{P} A$. Mais dans la preuve du théorème 3.7 , on a vu que $\Delta\left(\mathbf{2}^{\omega}\right) \not_{P} \check{D}_{0}$, et par 3.6, $\check{D}_{0}$ n'est pas $\operatorname{Pot}\left(\boldsymbol{\Sigma}_{1}^{0}\right)$; mais pour montrer que $\Delta\left(\mathbf{2}^{\omega}\right) \not_{P} \check{D}_{0}$, on a utilisé le fait que $D_{0}$ est à coupes co-dénombrables. Or il se trouve que les $\operatorname{Pot}\left(\boldsymbol{\Pi}_{1}^{0}\right)$ non $\operatorname{Pot}\left(\Sigma_{1}^{0}\right)$ à coupes co-dénombrables n'existent pas : sinon soit $\tau$ une topologie sur $Y$ rendant les coupes d'un tel $A$ fermées; $\Pi_{Y}^{\prime \prime}(\check{A})$ serait ouvert de $(Y, \tau)$ et on aurait $\Pi_{Y}^{\prime \prime}(\check{A})=\bigcup_{x \in X} \check{A}(x)=\bigcup_{n \in \omega} \check{A}\left(x_{n}\right)$ car $\Pi_{Y}^{\prime \prime}(\check{A})$ est un espace de Lindelöf; comme $\check{A}\left(x_{n}\right)$ est dénombrable, $\Pi_{Y}^{\prime \prime}(\check{A})$ le serait aussi et par la remarque $2.1, A$ serait $\operatorname{Pot}\left(\boldsymbol{\Delta}_{1}^{0}\right)$. On peut donc se demander si on n'a pas là une caractérisation des "vrais" $\operatorname{Pot}\left(\boldsymbol{\Pi}_{1}^{0}\right)$, à savoir : si $B \operatorname{est} \operatorname{Pot}\left(\boldsymbol{\Pi}_{1}^{0}\right)$, $B$ n'est pas $\operatorname{Pot}\left(\boldsymbol{\Sigma}_{1}^{0}\right)$ si et seulement si $\Delta\left(\mathbf{2}^{\omega}\right) \leq_{P} B$. On va voir que c'est bien le cas. On note $L_{0}:=\left\{(\alpha, \beta) \in \mathbf{2}^{\omega} \times \mathbf{2}^{\omega}: \alpha \leq_{\text {lex }} \beta\right\}$.

ThÉORÈme 4.13. Si A est $\operatorname{Pot}\left(D_{2}\left(\boldsymbol{\Sigma}_{1}^{0}\right)\right)$ dans un produit de deux espaces polonais, les conditions suivantes sont équivalentes:

(i) A n'est pas $\operatorname{Pot}\left(\boldsymbol{\Sigma}_{1}^{0}\right)$.

(ii) Il existe des fonctions injectives continues $\phi$, de $\mathbf{2}^{\omega}$ dans $X$, et $\psi$, de $\mathbf{2}^{\omega}$ dans $Y$, telles que $\Delta\left(\mathbf{2}^{\omega}\right)=(\phi \times \psi)^{-1}(A)$ ou $L_{0}=(\phi \times \psi)^{-1}(A)$.

Démonstration. Montrons que (ii) implique (i). On a vu au chapitre 2 que $\Delta\left(\mathbf{2}^{\omega}\right)$ n'est pas $\operatorname{Pot}\left(\boldsymbol{\Sigma}_{1}^{0}\right)$, donc si $\Delta\left(\mathbf{2}^{\omega}\right)=(\phi \times \psi)^{-1}(A), A$ n'est pas $\operatorname{Pot}\left(\boldsymbol{\Sigma}_{1}^{0}\right)$. Il suffit donc de voir que $L_{0}$ n'est pas $\operatorname{Pot}\left(\boldsymbol{\Sigma}_{1}^{0}\right)$, et il suffit de montrer que $\Delta\left(\mathbf{2}^{\omega}\right) \leq_{P} L_{0}$. Posons donc

$$
f(\alpha, \beta)= \begin{cases}(\alpha, \beta) & \text { si } \alpha \geq \beta \\ (\beta, \alpha) & \text { sinon. }\end{cases}
$$


Alors $\Delta\left(\mathbf{2}^{\omega}\right)=f^{-1}\left(L_{0}\right)$, et $f$ est dans $C_{0}$ :

$$
\begin{aligned}
f(\alpha, \beta) \in C \times D \Leftrightarrow & (\alpha \geq \beta \text { et } \alpha \in C \text { et } \beta \in D) \text { ou } \\
& (\alpha<\beta \text { et } \alpha \in D \text { et } \beta \in C) \\
\Leftrightarrow & (\alpha \in C \cap D \text { et } \beta \in C \cap D) \text { ou } \\
& (\alpha>\beta \text { et } \alpha \in C \text { et } \beta \in D) \text { ou } \\
& (\alpha<\beta \text { et } \alpha \in D \text { et } \beta \in C) .
\end{aligned}
$$

Montrons maintenant que (i) implique (ii) : $A$ est $\operatorname{Pot}\left(D_{2}\left(\boldsymbol{\Sigma}_{1}^{0}\right)\right)$, donc par la proposition 2.2 on a $A=\left(\bigcup_{n \in \omega} C_{n} \times D_{n}\right) \backslash V$, avec $C_{n}, D_{n}$ boréliens, $V$ dans $\operatorname{Pot}\left(\boldsymbol{\Sigma}_{1}^{0}\right)$, et la réunion disjointe.

En effet, on peut supposer cette réunion des rectangles disjointe : si $E=\bigcup_{n \in \omega} A_{n} \times B_{n}$, et si $I \subseteq n$, posons $C_{n, I}:=A_{n} \cap \bigcap_{i \in I}\left[A_{i} \backslash \bigcup_{j \in n \backslash I} A_{j}\right]$, $D_{n, I}:=B_{n} \backslash \bigcup_{i \in I} B_{i}$. Alors on vérifie facilement que $E=\bigcup_{n, I \subseteq n} C_{n, I} \times$ $D_{n, I}$, cette réunion étant disjointe.

On trouve alors $n$ tel que $\left(C_{n} \times D_{n}\right) \backslash V$ ne soit pas $\operatorname{Pot}\left(\boldsymbol{\Sigma}_{1}^{0}\right)$; or $\left(C_{n} \times D_{n}\right) \backslash V$ est $\operatorname{Pot}\left(\boldsymbol{\Pi}_{1}^{0}\right)$, donc si on admet le résultat pour $A$ dans $\operatorname{Pot}\left(\boldsymbol{\Pi}_{1}^{0}\right)$, on a le théorème, par disjonction de la réunion (en effet, $\phi$ (resp. $\psi$ ) est à valeurs dans $C_{n}\left(\operatorname{resp} . D_{n}\right)$ ).

Quitte à affiner les topologies, on peut supposer que $A$ est fermé, puisque la continuité de $\phi$ et $\psi$ avec des topologies plus fines entraînera la continuité avec les topologies initiales.

On construit par récurrence sur $|s|$, où $s \in 2^{<\omega}$, des ouverts-fermés non vides $V_{s} \subseteq X, W_{s} \subseteq Y$ tels que si $U_{s}:=V_{s} \times W_{s}$,

(i) $U_{s} \frown i \subseteq U_{s}$,

(ii) $\delta\left(U_{s}\right)<|s|^{-1}$ si $s \neq \emptyset$,

(iii) $V_{s \frown 0} \cap V_{s \frown 1}=W_{s \frown 0} \cap W_{s \frown 1}=\emptyset$,

(iv) $U_{s} \cap A \notin \operatorname{Pot}\left(\Sigma_{1}^{0}\right)$,

(v) $V_{s \frown 0} \times W_{s \frown 1} \subseteq \check{A}$.

Posons $U_{\emptyset}:=X \times Y$, et admettons avoir construit $\left(U_{s}\right)_{s \in 2 \leq n}$ vérifiant (i)-(v). Recouvrons $V_{s}$ et $W_{s}$ par des ouverts-fermés de diamètre au plus $(|s|+1)^{-1}$, soient $\left(V_{n}\right)$ et $\left(W_{p}\right)$. On a $A \cap U_{s}=\bigcup_{n, p \in \omega} A \cap\left(V_{n} \times W_{p}\right)$, donc l'un des $A \times\left(V_{n} \times W_{p}\right)$ n'est pas $\operatorname{Pot}\left(\boldsymbol{\Sigma}_{1}^{0}\right)$. Posons $V:=V_{n}, W:=W_{p}$; on a $U:=V \times W \subseteq U_{s}, A \cap U \notin \operatorname{Pot}\left(\Sigma_{1}^{0}\right)$, et $\delta(U)<(|s|+1)^{-1}$.

Posons $C:=\bigcup\left\{B: B \in \Sigma_{1}^{0}\left\lceil U\right.\right.$ et $\left.A \cap B \in \operatorname{Pot}\left(\Sigma_{1}^{0}\right)\right\}, A^{\prime}:=(A \cap U) \backslash C$. Comme $C$ est de Lindelöf, $A \cap C$ est $\operatorname{Pot}\left(\boldsymbol{\Sigma}_{1}^{0}\right)$.

Montrons qu'il existe $\left(x, x^{\prime}, y, y^{\prime}\right)$ dans $V^{2} \times W^{2}$ tels que $(x, y)$ et $\left(x^{\prime}, y^{\prime}\right)$ soient dans $A^{\prime}$ et $\left(x^{\prime}, y\right) \notin A$. Si tel n'est pas le cas, $\Pi_{X}^{\prime \prime} A^{\prime} \times \Pi_{Y}^{\prime \prime} A^{\prime} \subseteq U \cap A$, donc comme $V, W$, et $A$ sont fermés, $\overline{\Pi_{X}^{\prime \prime} A^{\prime}} \times \overline{\Pi_{Y}^{\prime \prime} A^{\prime}} \subseteq U \cap A$, d'où l'égalité $A \cap U=\left[\overline{\Pi_{X}^{\prime \prime} A^{\prime}} \times \overline{\Pi_{Y}^{\prime \prime} A^{\prime}}\right] \cup\left[A \cap\left(V \backslash \overline{\Pi_{X}^{\prime \prime} A^{\prime}} \times W\right)\right] \cup\left[A \cap\left(V \times W \backslash \overline{\Pi_{Y}^{\prime \prime} A^{\prime}}\right)\right]$. 
Le premier terme du membre de droite est un rectangle borélien, donc est $\operatorname{Pot}\left(\Sigma_{1}^{0}\right)$; le deuxième est contenu dans $C$, et vaut $A \cap C \cap\left[\left(V \backslash \overline{\Pi_{X}^{\prime \prime} A^{\prime}}\right) \times W\right]$, donc est $\operatorname{Pot}\left(\boldsymbol{\Sigma}_{1}^{0}\right)$, ainsi que le troisième. Donc $A \cap U$ est $\operatorname{Pot}\left(\boldsymbol{\Sigma}_{1}^{0}\right)$, ce qui est exclus.

Comme $U \backslash A$ est ouvert, soient $Z, T$ des ouverts tels que $\left(x^{\prime}, y\right)$ soit dans $Z \times T \subseteq U \backslash A$; on peut poser $V_{s \frown 0}:=Z, V_{s \frown 1}:=V \backslash Z, W_{s \frown 0}:=W \backslash T$, $W_{s \frown 1}:=T$.

Vérifions (iv) : $\left(x^{\prime}, y^{\prime}\right)$ est dans $U_{s \frown 0} \cap A=(Z \times W) \cap A$, donc $U_{s \frown 0} \cap A$ n'est pas $\operatorname{Pot}\left(\Sigma_{1}^{0}\right)$. De même pour $U_{s \frown 1} \cap A$, avec $(x, y)$.

Soit $\Phi$, de $\mathbf{2}^{\omega}$ dans $X \times Y$, définie par $\{\Phi(\alpha)\}=\bigcap_{n \in \omega} U_{\alpha\lceil n}$; alors on a $\Phi^{\prime \prime} \mathbf{2}^{\omega}=\operatorname{Gr}(f)$, où $f$ est une injection continue définie sur la copie $\Pi_{X}^{\prime \prime} \operatorname{Gr}(f)$ de $\mathbf{2}^{\omega}$. De plus $\operatorname{Gr}(f) \subseteq A$ car par (iv), il existe $\left(\gamma_{n}^{\alpha}, \beta_{n}^{\alpha}\right)$ dans $U_{\alpha\lceil n} \cap A$, et la suite $\left(\left(\gamma_{n}^{\alpha}, \beta_{n}^{\alpha}\right)\right)_{n \in \omega}$ converge vers $\Phi(\alpha)$, donc $\Phi(\alpha)$ est dans $A$ qui est fermé. Enfin, si $\alpha<_{\operatorname{lex}} \beta,\left(\left(\Pi_{X} \circ \Phi\right)(\alpha),\left(\Pi_{Y} \circ \Phi\right)(\beta)\right)$ n'est pas dans $A$, par (v). Posons donc $E:=\left(\left(\Pi_{X} \circ \Phi\right) \times\left(\Pi_{Y} \circ \Phi\right)\right)^{-1}(A)$; on a :

(a) $\Delta\left(\mathbf{2}^{\omega}\right) \subseteq E$

(b) $\alpha<_{\operatorname{lex}} \beta \Rightarrow(\alpha, \beta) \notin E$.

Montrons que $\mathbf{2}^{\omega}$ contient une copie $Z$ de $\mathbf{2}^{\omega}$ telle que $E \cap Z^{2}$ soit égal à $\Delta\left(\mathbf{2}^{\omega}\right) \cap Z^{2}$ ou à $L_{0} \cap Z^{2}$.

Premier cas : Pour toute suite de $2^{<\omega}, N_{s}^{2} \backslash E$ n'est pas antisymétrique. On construit alors par récurrence sur $|s|$ une suite $\left(V_{s}\right)$ d'ouvertsfermés non vides de $\mathbf{2}^{\omega}$ vérifiant :

(1) $V_{s}{ }_{i} \subseteq V_{s}$,

(2) $\delta\left(V_{s}\right)<|s|^{-1}$ si $s \neq \emptyset$,

(3) $\left(V_{s \frown 0} \times V_{s \frown 1}\right) \cup\left(V_{s \frown 1} \times V_{s \frown 0}\right) \subseteq \check{E}$.

Ceci ne pose aucun problème puisque $E$ est fermé dans $2^{\omega} \times \mathbf{2}^{\omega}$ et que $V_{s}^{2} \backslash E$ n'est pas antisymétrique. La formule $\{g(\alpha)\}:=\bigcap_{n \in \omega} V_{\alpha\lceil n}$ définit une injection continue $g$, qui est un homéomorphisme de $\mathbf{2}^{\omega}$ sur son image $Z$, qui par (a) vérifie $E \cap Z^{2}=\Delta\left(\mathbf{2}^{\omega}\right) \cap Z^{2}$.

Second cas: Il existe $s$ dans $2^{<\omega}$ telle que $N_{s}^{2} \backslash E$ soit antisymétrique. Alors par (a) et (b), $Z:=N_{s}$ vérifie $E \cap Z^{2}=L_{0} \cap Z^{2}$.

Soit alors $f$ un homéomorphisme décroissant de $\mathbf{2}^{\omega}$ sur $Z$; les fonctions composées $\phi:=\Pi_{X} \circ \Phi \circ f$ et $\psi:=\Pi_{Y} \circ \Phi \circ f$ répondent au problème.

Corollaire 4.14. (a) Sous les hypothèses du théorème 4.13, A n'est pas $\operatorname{Pot}\left(\boldsymbol{\Sigma}_{1}^{0}\right)$ si et seulement si $\Delta\left(\mathbf{2}^{\omega}\right) \leq_{P} A$.

(b) On ne peut pas trouver $A_{0}$ tel que si $A$ est borélien d'un produit de deux espaces polonais, on ait : A n'est pas $\operatorname{Pot}\left(\boldsymbol{\Pi}_{1}^{0}\right)$ si et seulement si $A_{0} \leq_{P} A$. 
(c) Soit $A$ un borélien d'un produit de deux espaces polonais. Alors $A$ est $\operatorname{Pot}\left(\boldsymbol{\Delta}_{1}^{0}\right)$ si et seulement si $A<_{P} \Delta\left(\mathbf{2}^{\omega}\right)$.

Démonstration. (a) Si $A$ n'est pas $\operatorname{Pot}\left(\boldsymbol{\Sigma}_{1}^{0}\right)$, par le théorème 4.13, $\Delta\left(\mathbf{2}^{\omega}\right) \leq_{P} A$ ou $L_{0} \leq_{P} A$, et on a vu dans la preuve que $\Delta\left(\mathbf{2}^{\omega}\right) \leq_{P} L_{0}$, donc $\Delta\left(\mathbf{2}^{\omega}\right) \leq_{P} A$. La réciproque résulte du chapitre 2 .

(b) Avec $A:=\check{\Delta}\left(\mathbf{2}^{\omega}\right)$, on voit que si $A_{0}$ existait, $A_{0}$ serait $\operatorname{Pot}\left(\boldsymbol{\Sigma}_{1}^{0}\right)$. Avec $A:=A_{0}$, on voit que $A_{0}$ serait $\operatorname{Pot}\left(\boldsymbol{\Sigma}_{1}^{0}\right)$ non $\operatorname{Pot}\left(\boldsymbol{\Pi}_{1}^{0}\right)$. Par (a), on aurait donc $\check{\Delta}\left(\mathbf{2}^{\omega}\right) \leq_{P} A_{0}$. Avec $A:=D_{0}$, on aurait $A_{0} \leq_{P} D_{0}$, donc $\Delta\left(\mathbf{2}^{\omega}\right) \leq_{P} \check{D}_{0}$, ce qui est exclus par la preuve du théorème 3.7.

(c) Si $A$ est $\operatorname{Pot}\left(\boldsymbol{\Delta}_{1}^{0}\right)$, et si

$$
f(x, y):= \begin{cases}\left(0^{\omega}, 0^{\omega}\right) & \text { si }(x, y) \in A, \\ \left(0^{\omega}, 1^{\omega}\right) & \text { sinon, }\end{cases}
$$

$f$ est dans $C_{0}$ et réduit $A$ à $\Delta\left(\mathbf{2}^{\omega}\right) ; \Delta\left(\mathbf{2}^{\omega}\right) \quad{ }_{P} A$ car $\Delta\left(\mathbf{2}^{\omega}\right)$ n'est pas $\operatorname{Pot}\left(\boldsymbol{\Sigma}_{1}^{0}\right)$.

Réciproquement, $A \leq_{P} \Delta\left(\mathbf{2}^{\omega}\right)$, donc $A$ est $\operatorname{Pot}\left(\boldsymbol{\Pi}_{1}^{0}\right)$; si $A$ n'était pas $\operatorname{Pot}\left(\boldsymbol{\Sigma}_{1}^{0}\right)$, par (a) on aurait $\Delta\left(\boldsymbol{2}^{\omega}\right) \leq_{P} A$, ce qui est exclus.

Le contre-exemple $\check{D}_{0}$ prouve que l'hypothèse " $A \in \operatorname{Pot}\left(D_{2}\left(\boldsymbol{\Sigma}_{1}^{0}\right)\right)$ " du théorème 4.13 et du corollaire 4.14(a) est optimale du point de vue classe de Wadge.

On ne peut pas se ramener à un seul exemple typique dans le théorème 4.13 , avec des réductions rectangulaires : si

$C \leq_{R} D \Leftrightarrow$ il existe $f, g$ boréliennes telles que $C=(f \times g)^{-1}(D)$, alors $\Delta\left(\mathbf{2}^{\omega}\right) \perp_{R} L_{0}$, comme on le vérifie immédiatement.

5. Uniformisation partielle des $G_{\delta}$. On va montrer dans ce paragraphe des théorèmes d'uniformisation partielle des $G_{\delta}$, dont le but est d'essayer de trouver une réciproque au lemme 3.5. Ces théorèmes sont à rapprocher d'une part de résultats dans $[\mathrm{GM}]$ et $[\mathrm{Ma}]$, où au lieu de considérer la catégorie, il est question d'ensembles de mesure 1 sur chacun des facteurs; et d'autre part de résultats de G. Debs et J. Saint Raymond, où il est question de fonctions totales et injectives, avec des hypothèses de compacité sur chacun des facteurs (cf. [D-SR]).

Lemme 5.1. Soient $X^{\prime}$ un ouvert-fermé non vide de $\omega^{\omega}, Y$ un espace polonais, $Y^{\prime}$ un ouvert non vide de $Y, \varepsilon>0$, et $O$ un ouvert dense de $X^{\prime} \times Y^{\prime}$ dont la projection est $X^{\prime}$; il existe des suites $\left(U_{k}\right)$ (d'ouvert-fermés non vides de $\left.\omega^{\omega}\right)$ et $\left(V_{k}\right)$ (d'ouverts non vides de $Y$ ) telles que:

(1) $\bigcup_{k \in \omega} U_{k}$ (resp. $\bigcup_{k \in \omega} \bar{V}_{k}$ ) est dense dans $X^{\prime}$ (resp. $\left.Y^{\prime}\right)$,

(2) $U_{k} \times V_{k} \subseteq O$,

(3) $\delta\left(U_{k}\right), \delta\left(V_{k}\right)<\varepsilon$, 
(4) $U_{p} \cap U_{q}=\emptyset$ si $p \neq q$.

Démonstration. Soit $\left(W_{m}\right)$ une partition de $X^{\prime}$ en ouvert-fermés, avec $\delta\left(W_{m}\right)<\varepsilon$ et $W_{m} \neq \emptyset$ (c'est possible car $X^{\prime}$ est homéomorphe à $\omega^{\omega}$ ). Soit $\left(T_{m}\right)$ une base de la topologie de $Y^{\prime}$. Par densité de $O$, on trouve $\left(x_{m}, y_{m}\right)$ dans $\left(W_{m} \times T_{m}\right) \cap O$, et un ouvert-fermé $X_{m}$ de $X^{\prime}$, un ouvert $Y_{m}$ de $Y^{\prime}$ tels que $\delta\left(Y_{m}\right)<\varepsilon$, et $\left(x_{m}, y_{m}\right) \in X_{m} \times \bar{Y}_{m} \subseteq\left(W_{m} \times T_{m}\right) \cap O$. Si $\bigcup_{m \in \omega} X_{m}$ est dense dans $X^{\prime}$, on a construit, en prenant $U_{k}:=X_{k}$ et $V_{k}:=Y_{k}$, les ouverts cherchés; en effet, ils vérifient bien (1)-(4).

Sinon, si $x \in X^{\prime} \backslash \overline{\bigcup_{m \in \omega} X_{m}}$, on trouve un ouvert-fermé $Z_{x}$ de $X^{\prime}, y_{x}$ dans $O(x)$, et un ouvert $R_{x}$ de $Y$ tels que l'on ait $\delta\left(Z_{x}\right), \delta\left(R_{x}\right)<\varepsilon$, et également l'inclusion $\left(x, y_{x}\right) \in Z_{x} \times \bar{R}_{x} \subseteq O \cap\left[\left(X^{\prime} \backslash \overline{\bigcup_{m \in \omega} X_{m}}\right) \times Y^{\prime}\right]$. On a

$$
X^{\prime} \backslash \overline{\bigcup_{m \in \omega} X_{m}}=\bigcup Z_{x}=\bigcup_{n \in \omega} Z_{x_{n}}=\bigcup_{n \in \omega}\left[Z_{x_{n}} \backslash \bigcup_{p<n} Z_{x_{p}}\right] \text {, }
$$

puisque l'espace $X^{\prime} \backslash \overline{\bigcup_{m \in \omega} X_{m}}$ est de Lindelöf. Posons $\widetilde{Z}_{n}:=Z_{x_{n}} \backslash$ $\bigcup_{p<n} Z_{x_{p}}$; s'il y a une infinité de $\widetilde{Z}_{n}$ non vides, on note $\left(Z_{n}\right)$ la suite formée de ces ouverts-fermés (on trouve donc $n_{k}$ tel que $Z_{k}=\widetilde{Z}_{n_{k}}$ ).

Sinon, on partitionne un $\widetilde{Z}_{n_{0}}$ non vide en une suite d'ouverts-fermés non vides de $X^{\prime}$, et on note $\left(Z_{n}\right)$ la suite formée des $\widetilde{Z}_{n}$ non vides (pour $n \neq n_{0}$ ) et de la partition de $\widetilde{Z}_{n_{0}}$; on a encore $Z_{k} \subseteq \widetilde{Z}_{n_{k}}$, avec éventuellement $n_{k}=n_{k^{\prime}}$.

Il reste à poser $R_{k}=R_{x_{n_{k}}}$, puis $U_{2 k}:=X_{k}, U_{2 k+1}:=Z_{k}, V_{2 k}:=Y_{k}$, $V_{2 k+1}:=R_{k}$.

ThÉORÈme 5.2. Soient $X, Y$ des espaces polonais non vides, $X$ étant parfait, $A$ un $G_{\delta}$ dense de $X \times Y$. Alors il existe des $G_{\delta}$ denses $F$ (dans $X)$ et $G($ dans $Y)$ et une surjection continue ouverte $f$ de $F$ sur $G$ dont le graphe est contenu dans $A$ (avec de plus $F$ homéomorphe à $\omega^{\omega}$ ).

Dém onstration. On peut supposer $A$ à coupes verticales co-maigres : $\check{A}$ est maigre, donc a ses coupes verticales maigres, sauf sur un ensemble maigre (théorème de Kuratowski-Ulam). $A$ a donc ses coupes verticales co-maigres sur un $G_{\delta}$ dense $H$ de $X$, qui comme $X$ est polonais parfait.

On peut supposer également que $X=\omega^{\omega}$ (de sorte que si $F$ est $G_{\delta}$ dense de $X, F$ est homéomorphe à $\omega^{\omega}$ ) : en effet, on procède comme dans la preuve du corollaire 1.7 .

Soit $\left(O_{n}\right)$ une suite d'ouverts denses de $X \times Y$ telle que $A=\bigcap_{n \in \omega} O_{n}$, $\phi_{0}$ de $\omega$ dans $\{\emptyset\}$ et, si $n>0, \phi_{n}$ une bijection de $\omega$ sur $\omega^{n}$. On construit une suite $\left(U_{s}\right)_{s \in \omega}<\omega$ d'ouverts-fermés non vides de $X$, et une suite $\left(V_{s}\right)_{s \in \omega<\omega}$ d'ouverts non vides de $Y$ vérifiant :

(i) $\bigcup_{n \in \omega} U_{s \frown n}\left(\operatorname{resp} . \bigcup_{n \in \omega} \bar{V}_{s \frown n}\right)$ est dense dans $U_{s}$ (resp. $\left.V_{s}\right)$, 
(ii) $U_{s} \times V_{s} \subseteq O_{|s|-1}$ si $s \neq \emptyset$,

(iii) $\delta\left(U_{s}\right), \delta\left(V_{s}\right)<|s|^{-1}$ si $s \neq \emptyset$,

(iv) $U_{s \frown n} \cap U_{s \frown m}=\emptyset$ si $n \neq m$,

(v) $\left(\bigcup_{k \in \omega} V_{\phi_{n}(k)}\right) \cap \bigcup_{m+p<n}\left[V_{\phi_{m}(p)} \backslash \bigcup_{l \in \omega} V_{\phi_{m}(p)-l}\right]=\emptyset$.

On pose $U_{\emptyset}:=\omega^{\omega}, V_{\emptyset}:=Y$; admettons avoir construit $\left(U_{s}\right)_{|s| \leq n}$ et $\left(V_{s}\right)_{|s| \leq n}$, ainsi que $\left(U_{\phi_{n}(p)-k}\right)_{p<m, k \in \omega},\left(V_{\phi_{n}(p)-k}\right)_{p<m, k \in \omega}$ vérifiant (i)-(v).

On construit, si ce n'est déjà fait, $\left(U_{\phi_{n}(m)-k}\right)_{k \in \omega},\left(V_{\phi_{n}(m)-k}\right)_{k \in \omega}$ en appliquant le lemme précédent à $\varepsilon:=(n+1)^{-1}$,

$$
X^{\prime}:=U_{\phi_{n}(m)}, \quad Y^{\prime}:=V_{\phi_{n}(m)} \backslash \bigcup_{q+p<n}\left[V_{\left.\phi_{q}(p) \backslash \bigcup_{l \in \omega} V_{\phi_{q}(p)-l}\right]},\right.
$$

et $O:=O_{n} \cap\left(X^{\prime} \times Y^{\prime}\right)$; les conditions demandées sont vérifiées, la densité ne posant pas de problème car l'adhérence ci-dessus est rare.

Posons $F:=\bigcap_{n \in \omega} \bigcup_{s \in \omega^{n}} U_{s}, G:=\bigcap_{n \in \omega} \bigcup_{s \in \omega^{n}} V_{s} ; F$ et $G$ sont clairement des $G_{\delta}$ denses de $X$ et $Y$.

Si $x$ est dans $F$, on trouve pour tout $n$ une unique suite $s_{n}$ de $\omega^{n}$ telle que $x \in U_{s_{n}}$ et aussi $s_{n} \prec s_{n+1}$; alors $\left(\bar{V}_{s_{n}}\right)$ définit $f(x)$ dans $G$, et $f$ est clairement continue car $f^{\prime \prime}\left(F \cap U_{s}\right) \subseteq G \cap V_{s}$. Montrons l'inclusion inverse, ce qui achèvera la preuve.

Soit donc $y$ dans $G \cap V_{s}$, et $p$ tel que $s=\phi_{|s|}(p)$; alors il existe un entier $\alpha(|s|)$ tel que $y \in V_{s \frown \alpha(|s|)}$, sinon $y \notin G$ car par (v), on aurait alors $y \notin \bigcup_{k \in \omega} V_{\phi_{|s|+p+1}(k)}$; on construit comme ceci par récurrence $\alpha$ dans $N_{s}$ tel que $\{y\}=\bigcap_{n \in \omega} V_{\alpha\lceil n}$; mais alors $\left(U_{\alpha\lceil n}\right)$ définit $x$ dans $F \cap U_{s}$ tel que $f(x)=y$.

On ne peut pas supprimer complètement une des hypothèses, ni espérer mieux avec ces hypothèses, dans le sens suivant :

Si on ne suppose pas $X$ parfait, prendre $X=\omega$ et $Y=\mathbf{2}^{\omega}$.

Si on ne suppose pas $A G_{\delta}$, prendre $X=Y=\mathbf{2}^{\omega}$ et

$$
A=\left(\mathbf{2}^{\omega} \times \mathbf{2}^{\omega}\right) \backslash\left(P_{\infty} \times P_{\infty}\right) .
$$

Si on ne suppose pas $A$ dense, prendre $X=Y=\mathbf{2}^{\omega}$ et $A=\left\{\left(0^{\omega}, 0^{\omega}\right)\right\}$.

On ne peut pas avoir $f$ totale ou surjective sur $Y$ : prendre $X=Y=\mathbf{2}^{\omega}$ et $A=P_{\infty}^{2}$.

Enfin, on ne peut pas avoir $f$ injective : prendre $X=\mathbf{2}^{\omega}$ et $Y=\omega$.

LEMME 5.3. Soient $\varepsilon>0, U$ et $V$ des ouverts non vides de $\omega^{\omega}$, et $O$ un ouvert dense de $U \times V$; on trouve alors des suites $\left(Z_{n}\right)$ et $\left(T_{n}\right)$ d'ouvertsfermés non vides de $\omega^{\omega}$ vérifiant:

(i) $\delta\left(Z_{n}\right), \delta\left(T_{n}\right)<\varepsilon$,

(ii) $Z_{n} \times T_{n} \subseteq O$,

(iii) $Z_{n} \cap Z_{m}=T_{n} \cap T_{m}=\emptyset$ si $n \neq m$,

(iv) $\bigcup_{n \in \omega} Z_{n}$ est dense dans $U$. 
Démonstration. Soient $\left(U_{n}\right)$ une base de la topologie de $U$ et $\left(V_{n}\right)$ une partition de $V$ en ouverts-fermés non vides de diamètre au plus $\varepsilon$. Alors $O \cap\left(U_{n} \times V_{n}\right)$ est non vide, donc contient $\left(x_{n}, y_{n}\right)$ et on trouve des suites $\left(X_{n}\right)$ et $\left(Y_{n}\right)$ d'ouverts-fermés de $\omega^{\omega}$, avec $\delta\left(X_{n}\right)<\varepsilon$ et $\left(x_{n}, y_{n}\right) \in X_{n} \times Y_{n} \subseteq O \cap\left(U_{n} \times V_{n}\right)$. Réduisons la suite $\left(X_{n}\right)$, ce qui fournit $\left(W_{n}\right)$. S'il y a une infinité de $W_{n}$ non vides, c'est terminé. Sinon, on partitionne un $W_{n_{0}}$ non vide et le $Y_{n_{0}}$ correspondant en une suite infinie d'ouverts-fermés non vides.

ThÉORÈme 5.4. Sous les hypothèses du théorème 5.2, si de plus $Y$ est parfait, on peut avoir pour $f$ un homéomorphisme.

Démonstration. Comme dans la preuve du théorème 5.2, on peut supposer que $X=Y=\omega^{\omega}$.

Soit $\left(O_{n}\right)$ une suite d'ouverts denses de $\omega^{\omega} \times \omega^{\omega}$ telle que $A=\bigcap_{n \in \omega} O_{n}$. On construit alors des suites d'ouverts-fermés non vides de $\omega^{\omega},\left(Z_{s}\right)_{s \in \omega<\omega}$ et $\left(T_{s}\right)_{s \in \omega<\omega}$, vérifiant :

(1) $\delta\left(Z_{s}\right), \delta\left(T_{s}\right)<|s|^{-1}$ si $s \neq \emptyset$,

(2) $Z_{s} \times T_{s} \subseteq O_{|s|-1}$ si $s \neq \emptyset$,

(3) $\bigcup_{n \in \omega} Z_{s \frown n}\left(\operatorname{resp} . T_{s}{ }_{n}\right)$ est dense dans $Z_{s}$ (resp. $T_{s}$ ),

(4) $Z_{s \frown n} \cap Z_{s \frown m}=T_{s \frown n} \cap T_{s \frown m}=\emptyset$ si $n \neq m$.

On pose pour commencer $Z_{\emptyset}=T_{\emptyset}:=\omega^{\omega}$. Admettons avoir construit $\left(Z_{s}\right)_{|s| \leq n}$ et $\left(T_{s}\right)_{|s| \leq n}$ vérifiant $(1)-(4)$.

On applique le lemme 5.3 à $\varepsilon:=(n+1)^{-1}, U:=Z_{s}, V:=T_{s}$, et $O:=O_{n} \cap(U \times V)$. Deux cas se présentent : si $T:=\bigcup_{m \in \omega} T_{m}$ est dense dans $T_{s}$, c'est terminé : on pose $Z_{s \frown m}:=Z_{m}$ et $T_{s \frown m}:=T_{m}$.

Sinon, on applique le lemme 5.3 à

$$
\begin{gathered}
O:=\left\{(x, y):(y, x) \in O_{n} \cap\left(Z_{s} \times\left(T_{s} \backslash \bar{T}\right)\right)\right\}, \\
U:=T_{s} \backslash \bar{T}, \quad V:=Z_{s}, \quad \varepsilon:=(n+1)^{-1},
\end{gathered}
$$

ce qui fournit $\left(Z_{m}^{\prime}\right)$ et $\left(T_{m}^{\prime}\right)$ vérifiant :

(i) $\delta\left(Z_{m}^{\prime}\right), \delta\left(T_{m}^{\prime}\right)<(n+1)^{-1}$,

(ii) $Z_{m}^{\prime} \times T_{m}^{\prime} \subseteq O_{n} \cap\left(Z_{s} \times\left(T_{s} \backslash \bar{T}\right)\right)$,

(iii) $\bigcup_{m \in \omega} T_{m}^{\prime}$ est dense dans $T_{s} \backslash \bar{T}$,

(iv) $Z_{p}^{\prime} \cap Z_{m}^{\prime}=T_{p}^{\prime} \cap T_{m}^{\prime}=\emptyset$ si $p \neq m$.

Posons, si $j \in \omega, n_{j}:=\min \left\{m \in \omega: Z_{j}^{\prime} \cap Z_{m} \neq \emptyset\right\}$; si $m \in \omega$,

$$
I_{m}:=\left\{j \in \omega: n_{j}=m\right\} \quad \text { et } \quad L_{m}:=\overline{\bigcup_{j \in I_{m}} Z_{j}^{\prime} \cap Z_{m}} .
$$

Quatre cas se présentent. 
Premier cas : $I_{m}=\emptyset$. On pose

$$
Z_{0}^{m}:=Z_{m}, \quad T_{0}^{m}:=T_{m}, \quad Z_{j}^{m}=T_{j}^{m}:=\emptyset \quad \text { si } j \geq 1 .
$$

Deuxième cas : $I_{m} \neq \emptyset$ et $Z_{m}=L_{m}$. Soit $j_{m}:=\min I_{m}$; on partitionne $Z_{j_{m}}^{\prime} \cap Z_{m}$ en deux ouverts-fermés non vides $\widetilde{Z}_{0}$ et $\widetilde{Z}_{1}$, et on pose

$$
\begin{gathered}
Z_{0}^{m}:=\widetilde{Z}_{0}, \quad T_{0}^{m}:=T_{m}, \\
Z_{1}^{m}:=\widetilde{Z}_{1}, \quad T_{1}^{m}:=T_{j_{m}}^{\prime} \\
Z_{j+1}^{m}:=Z_{m} \cap Z_{j}^{\prime}, \quad T_{j+1}^{m}:=T_{j}^{\prime} \quad \text { si } j>j_{m} \text { et } j \in I_{m}, \\
Z_{j}^{m}=T_{j}^{m}:=\emptyset \quad \text { si } j>1 \text { et }\left(j-1=j_{m} \text { ou } j-1 \notin I_{m}\right) .
\end{gathered}
$$

Troisième cas : $I_{m} \neq \emptyset$ est fini et $L_{m} \psi Z_{m}$. On pose

$$
\begin{gathered}
Z_{0}^{m}:=Z_{m} \backslash L_{m}, \quad T_{0}^{m}:=T_{m}, \\
Z_{j+1}^{m}:=Z_{m} \cap Z_{j}^{\prime}, \quad T_{j+1}^{m}:=T_{j}^{\prime} \quad \text { si } j \in I_{m}, \\
Z_{j}^{m}=T_{j}^{m}:=\emptyset \quad \text { si } j>1 \text { et } j-1 \notin I_{m} .
\end{gathered}
$$

Quatrième cas : $I_{m}$ est infini et $L_{m} \psi Z_{m}$. Soient $\psi$ une bijection de $\omega$ sur $I_{m}$, et $\left(\widetilde{Z}_{m}\right),\left(\widetilde{T}_{m}\right)$ des partitions en ouverts-fermés non vides de $Z_{m} \backslash L_{m}$ et $T_{m}$. On pose

$$
Z_{2 k}^{m}:=\widetilde{Z}_{2 k}, \quad T_{2 k}^{m}:=\widetilde{T}_{k}, \quad Z_{2 k+1}^{m}:=Z_{m} \cap Z_{\psi(k)}^{\prime}, \quad T_{2 k+1}^{m}:=T_{\psi(k)}^{\prime} .
$$

On renumérote, de façon à ce que

$$
\left\{Z_{s \frown p}: p \in \omega\right\}=\left\{Z_{j}^{i}: i, j \in \omega \text { et } Z_{j}^{i} \neq \emptyset\right\} ;
$$

on pose $T_{s \frown p}:=T_{j}^{i}$ si $Z_{s \frown p}=Z_{j}^{i}$, et $\left(Z_{s \frown p}\right),\left(T_{s \frown p}\right)$ répondent au problème, comme on le vérifie facilement.

Il est maintenant clair que $\bigcap_{n \in \omega} \bigcup_{s \in \omega^{n}}\left(Z_{s} \times T_{s}\right)$ est le graphe d'un homéomorphisme de $F:=\bigcap_{n \in \omega} \bigcup_{s \in \omega^{n}} Z_{s}$ sur $G:=\bigcap_{n \in \omega} \bigcup_{s \in \omega^{n}} T_{s}$.

Pour pouvoir appliquer ces résultats aux classes de Wadge potentielles, il faudrait traiter le cas où le $G_{\delta}$ est rare.

\section{Références}

[D-SR] G. Debs et J. Saint Raymond, Sélections boréliennes injectives, Amer. J. Math. 111 (1989), 519-534.

[GM] S. Graf and R. D. Mauldin, Measurable one-to-one selections and transition kernels, ibid. 107 (1985), 407-425.

[HKL] L. A. Harrington, A. S. Kechris and A. Louveau, A Glimm-Effros dichotomy for Borel equivalence relations, Amer. Math. Soc. 3 (1990), 903-928.

$[\mathrm{Ke}]$ A. S. Kechris, Measure and category in effective descriptive set theory, Ann. Math. Logic 5 (1973), 337-384.

[Ku] K. Kuratowski, Topology, Vol. 1, Academic Press, New York, 1966.

[Lo1] A. Louveau, livre à paraître. 
[Lo2] A. Louveau, Some results in the Wadge hierarchy of Borel sets, in: Cabal Seminar 79-81, A. S. Kechris, D. A. Martin and Y. N. Moschovakis (eds.), Lecture Notes in Math. 1019, Springer, 1983, 28-55.

[Lo3] -, A separation theorem for $\Sigma_{1}^{1}$ sets, Trans. Amer. Math. Soc. 260 (1980), 363-378.

[Lo4] -, Ensembles analytiques et boréliens dans les espaces produits, Astérisque 78 (1980).

[Lo-SR] A. Louveau and J. Saint Raymond, Borel classes and closed games: Wadgetype and Hurewicz-type results, Trans. Amer. Math. Soc. 304 (1987), 431-467.

[Ma] R. D. Mauldin, One-to-one selections, marriage theorems, Amer. J. Math. 104 (1982), 823-828.

[Mo] Y. N. Moschovakis, Descriptive Set Theory, North-Holland, 1980.

[P] T. C. Przymusi/nski, On the notion of n-cardinality, Proc. Amer. Math. Soc. 69 (1978), 333-338.

[W] W. W. Wadge, thesis, Berkeley, 1984.

EQUIPE D'ANALYSE

UNIVERSITÉ PARIS 6

BÔ̂TE 186

4, PLACE JUSSIEU

F-75 252 PARIS CEDEX 05, FRANCE 